\title{
Um problema inverso de identificação do coeficiente de condutividade da equação do calor envolvendo regiões não simplesmente conexas
}

\author{
Alexandre Kawano \\ TESE APRESENTADA \\ AO \\ Instituto DE MATEMÁtica E EstatísticA \\ DA \\ UNIVERSIDADE DE SÃo PAUlo \\ PARA \\ OBTENÇÃO DO TÍTULO DE DOUTOR \\ EM \\ CIÊNCIAS \\ Área de Concentração: Matemática Aplicada \\ Orientador: Prof. Dr. Paulo Domingos Cordaro
}

São Paulo, abril de 2007 


\section{Um problema inverso de identificação do coeficiente de condutividade da equação do calor envolvendo regiões não simplesmente conexas}

Este exemplar corresponde à redação final da tese devidamente corrigida e defendida por Alexandre Kawano e aprovada pela Comissão Julgadora.

São Paulo, 13 de abril de 2007.

Banca Examinadora:

Prof. Dr. Paulo Domingos Cordaro (orientador) - IME/USP

Prof. Dr. Clodoaldo Grotta Ragazzo - IME/USP

Prof. Dr. Raul Gonzalez Lima - POLI/USP

Prof. Dr. Gerson Petronilho - DM/USFSCAR

Prof. Dr. Francisco Gardel Leitão - DM/UFSC 


\section{Resumo}

Analisamos o problema inverso da identificação do coeficiente de condutividade $1+\rho$ da equação do calor.

Provamos um resultado de unicidade para uma versão linearizada desse problema em $\mathbb{R}^{n}$, para $n$ ímpar, que não depende da hipótese sobre a posição relativa entre o suporte, assumido compacto, da função desconhecida $\rho$ e um aberto limitado $\Omega^{*}$, onde as medidas de temperatura são efetuadas. Provamos o caso em que $\operatorname{supp}(\rho)$ pode ser não simplesmente conexo, e que $\Omega^{*}$ pode pertencer à uma de suas componentes limitadas.

Trata-se de uma extensão, para $n$ ímpar, de um teorema provado por Elayyan e Isakov. 


\begin{abstract}
We analyze the inverse problem of identifying the conductivity coefficient $1+\rho$ of the heat equation.

We prove an uniqueness result for a linearized version this problem in $\mathbb{R}^{\mathrm{n}}$ for $n$ odd that does not depend on hypothesis about the relative position of the support of the unknown function $\rho$, assumed to be compact, with respect to a bounded open set $\Omega^{*}$, where temperature measurements are taken. We proved a result in which $\operatorname{supp}(\Omega)$ can be not simply connected, and $\Omega^{*}$ can belong to one of its bounded connected components.

It is an extension, for $n$ odd, of a theorem proved by Elayyan and Isakov.
\end{abstract}




\section{Agradecimentos}

Tenho muitas razões para agradecer ao meu orientador, o Professor Paulo Domingos Cordaro. Algumas são relacionadas ao ofício da própria orientação, como por exemplo, a seleção de disciplinas importantes para a minha formação, e a observância de prazos para etapas a serem cumpridas. Entretanto, outras razões ultrapassam as fronteiras do puro dever para alcançar o domínio da extrema competência no trato da pessoa do orientando.

Aqui destaco apenas dois exemplos. Durante quatro semestres, o Professor Paulo assistiu semanalmente minhas apresentações de seminário, sempre me corrigindo e ensinando com paciência. Durante a preparação de um artigo submetido a uma revista e desta tese, ele me acompanhou quase que diariamente, fazendo correções e observações.

O Professor Paulo se fez para mim um modelo a ser seguido, que alterou minha atitude com relação aos meus próprios alunos. Passei a sentir maior responsabilidade pela formação acadêmica daqueles que de alguma forma possam depender de mim.

Agradeço também ao Professor Oscar Fortunato Vilcachagua Erazo, que durante um ano participou dos seminários acima citados, e aos docentes do IME que foram também meus professores em diversos cursos oferecidos pelo Instituto, os professores doutores: Alfredo Jorge Aragona Vallejo, Antonio Luiz Pereira, Clodoaldo Grota Ragazzo, Elisabeti Kira, Elói Medina Galego, Helena Maria Ávila de Castro, Ofélia Teresa Alas, Paolo Piccione, Roseli Fernandez, Sérgio Muniz Oliva, Trajano Couto Machado, Vera Lucia Carrara e Zara Issa Abud.

E por fim, não posso deixar de agradecer à Escola Politécnica da Universidade de São Paulo pelo apoio recebido. 


\section{Conteúdo}

1 Introdução

1.1 Enunciado de um problema de identificação de um coeficiente da Equação do

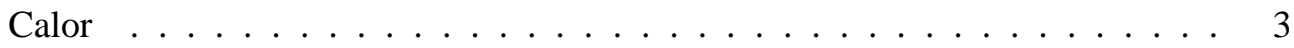

1.1.1 Linearização do problema . . . . . . . . . . . . . . 4

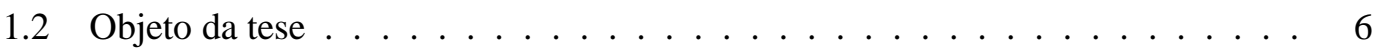

1.3 Plano geral do trabalho . . . . . . . . . . . . . . . 7

2 O resultado de Elayyan e Isakov $\quad 8$

2.1 Prova de unicidade para o problema . . . . . . . . . . . . 10

$3 \quad$ Extensão do resultado de Elayyan e Isakov $\quad 13$

3.1 Recapitulação importante sobre a determinação do coeficiente de condutividade da Equação do Calor . . . . . . . . . . . . . . . . . . . . . . 13

3.2 Proposta do problema a ser resolvido na tese . . . . . . . . . . . . . . . . . 14

3.3 Transformação do problema . . . . . . . . . . . . . . . . 15

3.3.1 Transformação da Equação do Calor em um problema hiperbólico . . 16

3.3.2 Solução do problema hiperbólico . . . . . . . . . . . . . . 24

3.4 Transformação do dado sintetizado em $\Gamma_{\Omega^{*}} \ldots \ldots \ldots \ldots$

3.5 Prova da unicidade para a recuperação de $\rho \ldots \ldots . \ldots . \ldots . \ldots 38$

3.5.1 O uso de médias esféricas . . . . . . . . . . . . . . . . 40

A Enunciados de alguns teoremas

A.1 Resultados da teoria dos espaços de Sobolev . . . . . . . . . . . . . . . . . . . . . . . 45

A.2 Alguns resultados para a Equação do Calor . . . . . . . . . . . . . . . 45

A.2.1 O conceito de solução fraca . . . . . . . . . . . . . 45

A.2.2 Espaços de solução . . . . . . . . . . . . . . . . . . . 46

A.2.3 Uma estimativa para a solução da Equação do Calor em função dos dados iniciais e de fronteira . . . . . . . . . . . . . . . 47 
A.3 Solução para a Equação da Onda não homogênea . . . . . . . . . . . . . . . . 49

A.4 Teorema de Paley Wiener . . . . . . . . . . . . . . . . 50

A.5 Teorema do Mínimo Módulo _ . . . . . . . . . . . . . . . . . . 51

A.6 Transformada de Laplace . . . . . . . . . . . . . . . . . . . . . . . 52

A.6.1 A transformada inversa . . . . . . . . . . . . . . . 53

A.6.2 Algumas propriedades . . . . . . . . . . . . 53 


\section{Capítulo 1}

\section{Introdução}

Problemas inversos se caracterizam pela busca da determinação das causas a partir das conseqüências, ou ainda dos parâmetros de um sistema, dado o par entrada/saída.

Comumente, um sistema físico é modelado por equações diferenciais ordinárias ou parciais, ou equações integrais; em geral o problema direto consiste na resolução delas, e o inverso, na determinação das condições de contorno ou iniciais, ou ainda de alguns dos coeficientes das equações.

Há quem sugira [11] que o problema inverso mais antigo de que se tem notícia é a estimação indireta do diâmetro da Terra através do tamanho de sombras por Eratóstenes em 200 AC. De lá para cá, muitas aplicações práticas importantes na indústria e na medicina que envolvem a determinação das causas a partir das conseqüências surgiram no mundo moderno. São exemplos a tomografia por raios-X e o tratamento de imagens em que se busca a versão mais nítida possível de uma foto borrada.

Paralelamente, e também inspiradas por algumas aplicações práticas, várias questões matemáticas foram formuladas, sendo uma delas aquela formulada por Marc Kac em 1966, "Can you hear the shape of a drum?" [8], de se perguntar se seria possível determinar-se univocamente a forma de um tambor a partir da informação de suas freqüências de ressonância. A resposta, negativa, para regiões em duas dimensões veio somente em 1991, como é contada em [2].

O problema a ser tratado na tese, a ser descrito nesta introdução (Seção 1.2) e com mais precisão no Capítulo 3, concerne a determinação de um coeficiente da Equação do Calor, mais precisamente o coeficiente de condutividade térmica do material suposto não constante espacialmente, conhecida a distribuição de temperatura em uma região do corpo para condições iniciais de temperatura bem definidas.

Mesmo que ele, o problema, tenha vida própria do ponto de vista estritamente matemático, gostaríamos de mencionar pelo menos uma aplicação industrial importante para a teoria 
desenvolvida nesse trabalho.

Em estruturas sujeitas à carregamentos cíclicos sucetíveis à falhas por fadiga, é imperativo que eventuais trincas sejam detectadas prematuramente (ver por exemplo [13], [9]). Assim, atualmente são usados métodos clássicos como o de correntes parasitas, raios-X e ultra-som, que necessitam varrer toda a extensão da estrutura.

A determinação da presença de rachaduras em materiais condutores de eletricidade é possível pelo método da tomografia por impedância elétrica [1] no qual a vantagem principal, quando comparado aos métodos clássicos, é que as medidas são feitas apenas na borda do membro estrutural sendo pesquisado. Entretanto, quando são usados materiais modernos como as cerâmicas, que são isolantes elétricos, o método da tomografia por impedância não funciona mais.

Uma solução é substituir o fenômeno elétrico e o modelo matemático associado, pelo fenômeno de condução térmica com sua Equação do Calor. O problema passa a ser a determinação do coeficiente de condutividade térmica ao longo do espaço: quando o valor determinado diferir significativamente do valor antecipado, isto é, supondo o corpo intacto, então possivelmente haverá a presença de uma trinca.

Mas a detecção da trinca somente será possível se a determinação do coeficiente de condutividade se der de modo unívoco. Propomos na tese a extensão de um teorema já publicado [3] que garante a unicidade sob algumas hipóteses.

\subsection{Enunciado de um problema de identificação de um coeficiente da Equação do Calor}

Sejam os abertos limitados não vazios $\Omega \subset \mathbb{R}^{n}$ com fronteira $\partial \Omega$ regular de classe $C^{2}$ por partes e $\Omega^{*} \subset \mathbb{R}^{n}$ tal que $\overline{\Omega^{*}} \bigcap \bar{\Omega}=\emptyset$. Sejam também um ponto $x_{0} \in \Omega^{*}$ e uma função $\rho: \mathbb{R}^{n} \rightarrow \mathbb{R}, \rho \in \mathrm{H}^{2 m+1}\left(\mathbb{R}^{n}\right)$, com suporte $\operatorname{supp}(\rho) \subset \Omega ; 2 m \geq[n / 2], 2 m \in \mathbb{Z}_{+}$, em que $[x]$ retorna o maior número inteiro menor ou igual a $x$ que o aproxima. Considere agora uma solução $u_{x_{0}}$ da equação

$$
\begin{cases}\frac{\partial u_{x_{0}}}{\partial t}-\operatorname{div}\left((1+\rho) \vec{\nabla} u_{x_{0}}\right)=\delta \otimes \delta_{x_{0}}, & \text { em }[0, T] \times \mathbb{R}^{n} \\ u_{x_{0}}=0, & \text { em }\{0\} \times \mathbb{R}^{n} .\end{cases}
$$

O problema é recuperarmos a função $\rho$ dado o conhecimento sintetizado no conjunto

$$
\Gamma_{\Omega^{*}}=\left\{u_{x}(T, x) \mid x \in \Omega^{*}\right\} .
$$

Fisicamente, $\Gamma_{\Omega^{*}}$ incorpora a informação sobre a temperatura no instante $T$, em cada ponto $x \in \Omega^{*}$ após a aplicação de uma carga térmica $\delta \otimes \delta_{x}$ aplicada no mesmo ponto $x$, em $t=0$. 
Observação 1.1. O fato da solução do problema inverso $\rho$ estar no espaço $\mathrm{H}^{2 m+1}\left(\mathbb{R}^{n}\right)$, com $2 m \geq[n / 2], 2 m \in \mathbb{Z}_{+}$, faz com que faça sentido tomar-se valores de $u_{x}$ em cada ponto $(T, x)$, como justificaremos adiante na Proposição 1.1.

O problema é não-linear e muito instável numericamente. Em busca de uma solução aproximada para o problema, Elayyan e Isakov [3] sugerem sua linearização.

Para a prova de que a equação linearizada se aproxima da equação dada, precisamos de um teorema, o Teorema A.4 enunciado no apêndice, para uma estimativa da solução da Equação do Calor em função dos dados iniciais e de fronteira.

\subsubsection{Linearização do problema}

Interpretamos $\rho \in \mathrm{H}^{2 m+1}(\Omega), \operatorname{supp}(\rho) \subset \Omega$ como uma perturbação em torno da unidade para a formação do coeficiente $1+\rho$ da Equação (1.1).

Denotamos por $\dot{u}_{x_{0}}$ a solução do problema não perturbado:

$$
\begin{cases}\frac{\partial}{\partial t} \dot{u}_{x_{0}}-\operatorname{div} \vec{\nabla} \dot{u}_{x_{0}}=\delta \otimes \delta_{x_{0}}, & \text { em }[0, T] \times \mathbb{R}^{n} \\ \dot{u}_{x_{0}}=0, & \text { em }\{0\} \times \mathbb{R}^{n} \backslash\left\{x_{0}\right\}\end{cases}
$$

Sabe-se que a solução de (1.3) é dada por

$$
\dot{u}_{x_{0}}= \begin{cases}\frac{1}{(\sqrt{4 \pi t})^{n}} \exp \left[-\frac{\left\|x-x_{0}\right\|^{2}}{4 t}\right], & t>0 \\ 0, & t \leq 0\end{cases}
$$

Chamamos de $u_{x_{0}, \rho}$ a solução do problema do calor com a perturbação $\rho$ :

$$
\begin{cases}\frac{\partial}{\partial t} u_{x_{0}, \rho}-\operatorname{div}\left((1+\rho) \vec{\nabla} u_{x_{0}, \rho}\right)=\delta \otimes \delta_{x_{0}}, & \text { em }[0, T] \times \mathbb{R}^{n} \\ u_{x_{0}, \rho}=0 & , \text { em }\{0\} \times \mathbb{R}^{n} \backslash\left\{x_{0}\right\} .\end{cases}
$$

Usando (1.3) e (1.5), obtemos uma equação para $v_{x_{0}, \rho} \doteq u_{x_{0}, \rho}-\dot{u}_{x_{0}}$ :

$$
\begin{cases}\frac{\partial}{\partial t} v_{x_{0}, \rho}-\Delta v_{x_{0}, \rho}=\operatorname{div}\left(\rho \vec{\nabla} v_{x_{0}, \rho}\right)+\operatorname{div}\left(\rho \vec{\nabla} \dot{u}_{x_{0}}\right), & \text { em }[0, T] \times \mathbb{R}^{n} \\ v_{x_{0}, \rho}=0, & \text { em }\{0\} \times \mathbb{R}^{n} \backslash\left\{x_{0}\right\} .\end{cases}
$$

Podemos ainda manipular (1.6) para obter uma equação equivalente:

$$
\begin{cases}\frac{\partial}{\partial t} v_{x_{0}, \rho}-\operatorname{div}\left((1+\rho) \vec{\nabla} v_{x_{0}, \rho}\right)=\operatorname{div}\left(\rho \vec{\nabla} \dot{u}_{x_{0}}\right), & \text { em }[0, T] \times \mathbb{R}^{n} \\ v_{x_{0}, \rho}=0, & \text { em }\{0\} \times \mathbb{R}^{n} \backslash\left\{x_{0}\right\} .\end{cases}
$$


Basta resolver (1.7) para obtermos $v_{x_{0}, \rho}$, e a partir dela, conhecendo-se $\dot{u}_{x_{0}}$, temos

$$
u_{x_{0}, \rho}=v_{x_{0}, \rho}+\dot{u}_{x_{0}} .
$$

É necessário verificarmos se faz sentido tomar-se a temperatura em um ponto em um instante $t>0$ determinado.

Proposição 1.1. A função $v_{x_{0}, \rho}$ é contínua nas variáveis temporal e espacial.

Prova: No problema (1.7), faça $h \doteq \operatorname{div}\left(\rho \vec{\nabla} \dot{u}_{x_{0}}\right)$. Como $\bar{\Omega} \cap \bar{\Omega}^{*}=\emptyset$, a regularidade de $h$ depende somente da regularidade de $\rho$, isto é, como $\rho \in \mathrm{H}^{2 m+1}\left(\mathbb{R}^{\mathrm{n}}\right)$, temos que

$$
\frac{\mathrm{d}^{k} h}{\mathrm{~d} t^{k}} \in \mathrm{L}^{2}\left(0, T ; \mathrm{H}^{2 m}\left(\mathbb{R}^{\mathrm{n}}\right)\right), \text { para } k=0, \ldots, m \text {. }
$$

Pela Proposição A.3, temos

$$
\begin{aligned}
& v_{x_{0}, \rho} \in \mathrm{L}^{2}\left(0, T ; \mathrm{H}_{l o c}^{2 m+2}\left(\mathbb{R}^{\mathrm{n}}\right)\right), \\
& v_{x_{0}, \rho}^{\prime} \in \mathrm{L}^{2}\left(0, T ; \mathrm{H}_{l o c}^{2 m}\left(\mathbb{R}^{\mathrm{n}}\right)\right) .
\end{aligned}
$$

Pela Proposição A.1,

$$
v_{x_{0}, \rho} \in \mathcal{C}^{0}\left([0, T], \mathrm{H}_{l o c}^{2 m+1}\left(\mathbb{R}^{\mathrm{n}}\right)\right) .
$$

Como por hipótese $2 m+1 \geq[n / 2]+1$, pela Proposição A.2 $v_{x_{0}, \rho}$ é contínua nas variáveis temporal e espacial.

Resolvendo a Equação (1.7) para cada $\rho \in \mathrm{H}^{2 m+1}\left(\mathbb{R}^{\mathrm{n}}\right), \operatorname{supp}(\rho) \subset \Omega$, temos um operador $F, \rho \stackrel{F}{\mapsto} v_{x_{0}, \rho} \subset \mathcal{C}^{0}\left([0, T], \mathrm{L}_{l o c}^{2}\left(\mathbb{R}^{\mathrm{n}}\right)\right)$ que é não linear. Note que $\rho$ é a incógnita do problema inverso.

Suprimindo o primeiro termo do lado direito da Equação (1.6), obteremos uma equação em $v_{x_{0}}$, que resolvida para cada $\rho$, fornece um operador linear $\rho \mapsto v_{x_{0}}$.

$$
\begin{cases}\frac{\partial}{\partial t} v_{x_{0}}-\Delta v_{x_{0}}=\operatorname{div}\left(\rho \vec{\nabla} \dot{u}_{x_{0}}\right) & , \text { em }[0, T] \times \mathbb{R}^{n} \\ v_{x_{0}}=0 & , \text { em }\{0\} \times \mathbb{R}^{n} .\end{cases}
$$

Observação 1.2. Se a Equação (1.5) for usada para definir um operador $\rho \stackrel{G}{\mapsto} u_{x_{0}, \rho}$, então (1.8) corresponde à derivada de Gateaux de $G$.

É claro que devemos provar que em algum sentido razoável a aproximação obtida por essa linearização do problema é válida. Se a diferença entre $v_{x_{0}, \rho}$ e $v_{x_{0}}$ for pequena, então $\dot{u}_{x_{0}}+v_{x_{0}}$ aproxima $u_{x_{0}, \rho}$, que é a solução exata do problema perturbado (1.5).

Podemos ver que $\rho$ é contínua pelo Teorema do Mergulho de Sobolev. Esse teorema afirma que $\mathrm{H}^{s}\left(\mathbb{R}^{\mathrm{n}}\right) \hookrightarrow \mathcal{C}\left(\mathbb{R}^{\mathrm{n}}\right)$ quando $s>n / 2$. Como $s=2 m+1 \geq[n / 2]+1>n / 2-1+1=n / 2$, vemos que de fato, $\rho$ é mesmo contínua. 
Lema 1.2. Suponha que $\|\rho\|_{L^{\infty}(\Omega)}<\epsilon$. Então existe $C>0$ independente de $\epsilon$ tal que

$$
\left|v_{x_{0}}-v_{x_{0}, \rho}\right|_{[0, T] \times \mathbb{R}^{\mathrm{n}}}<C \epsilon^{2} .
$$

Prova: Inicialmente, mostremos que $\left|v_{x_{0}, \rho}\right|_{\left.\mathbb{R}^{n} \times\right] 0, T[}<C \epsilon$.

Podemos limitar o termo do lado direito de (1.7), lembrando que como $x_{0} \in \Omega^{*} \mathrm{e} \overline{\Omega^{*}} \cap \bar{\Omega}=$ $\emptyset$, $\dot{u}_{x_{0}}$ dada por (1.4) é $\mathcal{C}^{\infty}$ em $\bar{\Omega} \times[0, T]$. Além disso, $\rho$ é nula fora de $\Omega$. Assim, $\exists C>0$ tal que

$$
\left\|\rho \vec{\nabla} \dot{u}_{x_{0}}\right\|_{L^{2}\left([0, T] \times \mathbb{R}^{n}\right)}<C \epsilon .
$$

Agora, usando o Teorema A.4, temos, após renomear a constante $C$ :

$$
\left|v_{x_{0}, \rho}\right|_{[0, T] \times \mathbb{R}^{n}} \leq C\left\|\rho \vec{\nabla} \dot{u}_{x_{0}}\right\|_{L^{2}\left([0, T] \times \mathbb{R}^{n}\right)}<C \epsilon .
$$

Para mostrar que $\left|v_{x_{0}}-v_{x_{0}, \rho}\right|_{\mathbb{R}^{n} \times[0, T]}<C \epsilon^{2}$, comparamos a equação não linearizada (1.6) com a linearizada (1.8) para obter

$$
\begin{cases}\frac{\partial}{\partial t}\left(v_{x_{0}, \rho}-v_{x_{0}}\right)-\triangle\left(v_{x_{0}, \rho}-v_{x_{0}}\right)= & , \text { em }[0, T] \times \mathbb{R}^{n} \\ \operatorname{div}\left(\rho \vec{\nabla} v_{x_{0}, \rho}\right) & , \text { em }\{0\} \times \mathbb{R}^{n} .\end{cases}
$$

Agora, usando a estimativa (1.9) e novamente o Teorema A.4, temos

$$
\begin{aligned}
\left\|v_{x_{0}, \rho}-v_{x_{0}}\right\|_{[0, T] \times \mathbb{R}^{n}} & \leq C\left\|\rho \vec{\nabla} v_{x_{0}, \rho}\right\|_{\mathrm{L}^{2}\left([0, T] \times \mathbb{R}^{n}\right)} \\
& <C \epsilon\left\|\vec{\nabla} v_{x_{0}, \rho}\right\|_{\mathrm{L}^{2}\left([0, T] \times \mathbb{R}^{n}\right)}<C \epsilon\left|v_{x_{0}, \rho}\right|_{[0, T] \times \mathbb{R}^{n}}<C \epsilon^{2} .
\end{aligned}
$$

Tendo concluído que a solução da equação linearizada aproxima a Equação (1.6), passamos a resolver o problema da identificação de $\rho$ em (1.8), que é o objeto de estudo da tese.

\subsection{Objeto da tese}

Elayyan e Isakov [3] provam que o dado

$$
\Gamma_{\Omega^{*}}=\left\{v_{x}(T, x) \mid x \in \Omega^{*}\right\}
$$

é suficiente para identificar univocamente $\rho$ na equação

$$
\begin{cases}\frac{\partial}{\partial t} v_{x_{0}}-\Delta v_{x_{0}}=\operatorname{div}\left(\rho \vec{\nabla} \dot{u}_{x_{0}}\right) & , \text { em }[0, T] \times \mathbb{R}^{n}, \\ v_{x_{0}}=0 & , \text { em }\{0\} \times \mathbb{R}^{n},\end{cases}
$$


quando $\overline{\Omega^{*}}$ está contido na componente ilimitada de $\complement \bar{\Omega}$.

No presente trabalho, estenderemos o resultado removendo essa restrição topológica para o caso da dimensão espacial ser ímpar.

O Teorema de Elayyan e Isakov é ainda enunciado em [6] na versão restrita.

\subsection{Plano geral do trabalho}

No capítulo seguinte, apresentamos formalmente o teorema provado por Elayyan e Isakov, em cuja demonstração propriedades de funções analíticas reais e o Teorema de Paley-Wiener são empregados. No Capítulo 3, propomos a extensão do resultado de Elayyan e Isakov, enunciando-a no Teorema 3.1. Sua prova envolve a transformação da Equação do Calor em um problema hiperbólico e a utilização de médias esféricas. 


\section{Capítulo 2}

\section{O resultado de Elayyan e Isakov}

Após ter feita a linearização do problema no capítulo anterior, apresentamos agora o problema resolvido por Elayyan e Isakov em [3].

Teorema 2.1. Sejam: um aberto $\Omega \subset \mathbb{R}^{\mathrm{n}}$ limitado não vazio, sendo $W$ a componente conexa ilimitada de $\complement \bar{\Omega}, \Omega^{*} \subset \mathbb{R}^{\mathrm{n}}$ limitado não vazio tal que $\overline{\Omega^{*}} \subset W$, e uma função $\rho: \mathbb{R}^{n} \rightarrow \mathbb{R}$, $\rho \in \mathrm{L}^{\infty}\left(\mathbb{R}^{n}\right), \operatorname{com} \operatorname{supp}(\rho) \subset \Omega$.

Considere agora a Equação (1.8 repetida):

$$
\begin{cases}\frac{\partial}{\partial t} v_{x_{0}}-\Delta v_{x_{0}}=\operatorname{div}\left(\rho \vec{\nabla} \dot{u}_{x_{0}}\right) & , \text { em }[0, T] \times \mathbb{R}^{n} \\ v_{x_{0}}=0 & , \text { em }\{0\} \times \mathbb{R}^{n}\end{cases}
$$

A solução da equação é $v_{x_{0}}(t, x)$.

$O$ conhecimento sintetizado no conjunto

$$
\Gamma_{\Omega^{*}}=\left\{v_{x}(T, x) \mid x \in \Omega^{*}\right\}
$$

recupera univocamente a função $\rho$.

Observação 2.1. O espaço da função $\rho$, que tem suporte em $\Omega$, foi alterado de $\mathrm{H}^{2 m+1}\left(\mathbb{R}^{\mathrm{n}}\right)$, usado nas seções anteriores, para $\mathrm{L}^{\infty}(\Omega)$. A restrição anterior havia sido colocada para garantir que a solução da Equação do Calor resultasse em uma função contínua, de modo a fazer sentido físico a medida da temperatura em um ponto. Entretanto, para o problema linearizado, mesmo que $\rho \in \mathrm{L}^{\infty}(\Omega)$, a solução $v_{x_{0}}(t, x)$ é contínua nos pontos onde ela será medida, como veremos através de sua fórmula explícita.

Observação 2.2. A distribuição que aparece no lado direito da equação do Teorema 2.1, $\operatorname{div}\left(\rho \vec{\nabla} \dot{u}_{x_{0}}\right)$, faz sentido quando atua em funções teste $\phi \in \mathcal{C}_{c}^{\infty}\left([0,+\infty) \times \mathbb{R}^{\mathrm{n}}\right)$. 
A ação de $\operatorname{div}\left(\rho \vec{\nabla} \dot{u}_{x_{0}}\right)$ em $\phi$ pode ser compreendida através da seguinte manipulação, que se vale da definição da operação da derivação de distribuições:

$$
\begin{aligned}
\int_{0}^{+\infty} \int_{\mathbb{R}^{\mathrm{n}}} \operatorname{div}\left(\rho \vec{\nabla} \dot{u}_{x_{0}}\right)(t, x) \phi(t, x) \mathrm{d} x \mathrm{~d} t= \\
=\sum_{j=1}^{n} \int_{0}^{+\infty} \int_{\mathbb{R}^{\mathrm{n}}} \frac{\partial}{\partial x_{j}}\left(\rho \frac{\partial \dot{u}_{x_{0}}}{\partial x_{j}}\right)(t, x) \phi(t, x) \mathrm{d} x \mathrm{~d} t= \\
=-\int_{0}^{+\infty} \int_{\mathbb{R}^{\mathrm{n}}} \rho(x) \vec{\nabla} \dot{u}_{x_{0}}(t, x) \circ \vec{\nabla} \phi(t, x) \mathrm{d} x \mathrm{~d} t .
\end{aligned}
$$

Elayyan e Isakov solucionaram o problema da identificação de $\rho$ convertendo-o em uma equação de convolução. Analisemos seus passos.

Usando a solução fundamental da Equação do Calor

$$
G(t, x)= \begin{cases}\frac{1}{(4 \pi t)^{n / 2}} \exp \left[\left(-\frac{|x|^{2}}{4 t}\right)\right] ; & t>0, x \in \mathbb{R}^{n} \\ 0 ; & t \leq 0\end{cases}
$$

a solução de (1.8) pode ser expressa por

$$
v_{x_{0}}(t, x)=-\int_{0}^{t} \int_{\Omega} \rho(y) \vec{\nabla} G(x-y, t-\tau) \circ \vec{\nabla} \dot{u}_{x_{0}}(y, \tau) \mathrm{d} y \mathrm{~d} \tau .
$$

Substituindo as fórmulas para $\dot{u}_{x_{0}}$ e $G$, respectivamente (1.4) e (2.1) em (2.2), temos:

$$
\begin{aligned}
v_{x_{0}}(t, x)=- & \frac{1}{4^{n+1} \pi^{n}} \\
& \int_{0}^{t} \int_{\Omega} \rho(y) \frac{(x-y) \circ\left(x_{0}-y\right)}{((t-\tau) \tau)^{n / 2+1}} \exp \left[-\frac{|x-y|^{2}}{4(t-\tau)}-\frac{\left|x_{0}-y\right|^{2}}{4 \tau}\right] \mathrm{d} y \mathrm{~d} \tau .
\end{aligned}
$$

Por compatibilidade com o dado (1.2) do problema inverso, colocamos o sensor na mesma posição da fonte de calor, isto é, fazemos $x=x_{0}$, e fixamos $t=T$. Dai, a distribuição de temperatura em $x \in \Omega^{*}$ é dada por

$$
\begin{aligned}
& v_{x}(T, x)=-\frac{1}{4^{n+1} \pi^{n}} \\
& \quad \int_{0}^{T} \int_{\Omega} \rho(y) \frac{|x-y|^{2}}{((T-\tau) \tau)^{n / 2+1}} \exp \left[-\frac{|x-y|^{2} T}{4(T-\tau) \tau}\right] \mathrm{d} y \mathrm{~d} \tau .
\end{aligned}
$$

Para simplificar a notação, chamamos

$$
v(t, x) \doteq v_{x}(t, x)
$$

O problema inverso passa a ser encarado como: Dado o conhecimento de $v(T, x)$ para $x \in \Omega^{*}$, determinar $\rho \in \mathrm{L}^{\infty}, \operatorname{supp}(\rho) \subset \Omega$ tal que satisfaça

$$
A \rho=v,
$$


em que

$$
A \rho(x)=k * \rho=\int_{\Omega} k(x-y) \rho(y) \mathrm{d} y, x \in \Omega^{*}
$$

$$
k(x)=-\frac{1}{4^{n+1} \pi^{n}} \int_{0}^{T} \frac{|x|^{2}}{((T-\tau) \tau)^{n / 2+1}} \exp \left[-\frac{|x|^{2} T}{4(T-\tau) \tau}\right] \mathrm{d} \tau .
$$

Para a prova da unicidade do problema proposto, usaremos a Transformada de Fourier e o conhecimento de fatos sobre o crescimento de funções holomorfas. Precisaremos do Teorema de Paley-Wiener (Teorema A.5) e de um resultado conhecido como o Teorema do Mínimo Módulo (Teorema A.6).

Agora estamos em condições de provar a unicidade para o Problema (2.5), isto é supondo que existe alguma solução $\rho$, ela é única.

\subsection{Prova de unicidade para o problema}

Teorema 2.2 (Unicidade). Seja $F=\left\{\rho \in \mathrm{L}^{\infty}\left(\mathbb{R}^{n}\right) \mid \operatorname{supp}(\rho) \subset \Omega\right\}$. Dada $v \in A(F), A$ descrito em (2.6), existe somente uma função $\rho \in F$, tal que a equação

$$
A \rho(x)=v(x), \forall x \in \Omega^{*}
$$

seja satisfeita.

Observação 2.3. Lembre-se de que $\Omega \subset \mathbb{R}^{n}$ é aberto limitado não vazio, sendo $W$ a componente conexa ilimitada de $\complement \bar{\Omega} . \Omega^{*} \subset \mathbb{R}^{n}$ é outro aberto limitado não vazio tal que $\overline{\Omega^{*}} \subset W$.

Dada a natureza do operador $A$, a função $v$ é real analítica em $\mathbb{R}^{n} \backslash \Omega$. Assim, se $v$ se anular em um aberto de $\mathbb{R}^{n} \backslash \Omega$, v será automaticamente nula na componente conexa que contiver esse aberto. Em particular, tomaremos esse aberto como sendo $\Omega^{*}$ e provaremos que necessariamente $\rho \equiv 0$.

Preparando a prova do teorema, tomemos a transformada de Fourier de (2.6), ou seja, de $k * \rho=v:$

$$
\hat{k} \hat{\rho}=\hat{v}
$$

A transformada de Fourier de $k$,

$$
\hat{k}(\xi)=\int_{\mathbb{R}^{\mathrm{n}}} k(x) \exp [-\imath x \xi] \mathrm{d} x
$$

é facilmente calculada lembrando que para qualquer $w \in \mathcal{S}^{\prime}, \mathcal{S}^{\prime}$ sendo o dual do espaço das funções rapidamente decrescentes de Schwartz (ver p.ex. [5]), vale $\widehat{x_{j} w}=\imath \partial_{\xi_{j}} \widehat{w}(\xi)$, e dai:

$$
\widehat{x^{2} w}=-\triangle \widehat{w}
$$


Assim, como a transformada de Fourier de $w(x)=\exp \left[-\frac{\lambda|x|^{2}}{2}\right], \lambda>0$, é

$$
\widehat{w}(\xi)=\frac{(2 \pi)^{n / 2}}{\lambda^{n / 2}} \exp \left[-\frac{|\xi|^{2}}{2 \lambda}\right]
$$

temos

$$
\mathcal{F}\left(|x|^{2} \exp \left[-\frac{\lambda|x|^{2}}{2}\right]\right)=-\frac{(2 \pi)^{n / 2}}{\lambda^{n / 2+1}}\left(\frac{|\xi|^{2}}{\lambda}-n\right) \exp \left[-\frac{|\xi|^{2}}{2 \lambda}\right] .
$$

Agora a transformada de Fourier de $k$ pode ser calculada:

$$
\widehat{k}(\xi)=\int_{\mathbb{R}^{n}} \underbrace{-\frac{1}{4(4 \pi)^{n}} \int_{0}^{T} \frac{|x|^{2}}{((T-\tau) \tau)^{n / 2+1}} \mathrm{e}^{-\frac{|x|^{2} T}{4(T-\tau) \tau}} \mathrm{d} \tau}_{k(x)} \mathrm{e}^{-\imath x \xi} \mathrm{d} x .
$$

Aplicando o Teorema de Tonelli para trocar a ordem de integração e usando (2.8) com

$$
\lambda=\frac{T}{2 \tau(T-\tau)},
$$

temos:

$$
\widehat{k}(\xi)=c_{n} \int_{0}^{T}\left(\frac{2 \tau(T-\tau)}{T}|\xi|^{2}-n\right) \exp \left[-|\xi|^{2} \frac{\tau(T-\tau)}{T}\right] \mathrm{d} \tau,
$$

em que

$$
c_{n}=\frac{1}{2^{n+1} \pi^{n / 2} T^{n / 2+1}} .
$$

Lema 2.3 (Minoração de $|\hat{k}|$ ). Dado $\xi_{0} \in \mathbb{R}^{n}$ não nulo, existe $\epsilon>0$ tal que para todo $\zeta$ da forma $\zeta=\imath R \xi_{0}$, em que $R>0$, vale a desigualdade

$$
|\hat{k}(\zeta)| \geq \epsilon \exp \left[\epsilon R^{2}\left|\xi_{0}\right|^{2}\right]
$$

Prova: Basta substituir $\zeta=\imath R \xi_{0}$ no lugar de $\xi$ na Fórmula (2.9).

$$
\widehat{k}(\zeta)=c_{n} \int_{0}^{T}\left(-\frac{2 \tau(T-\tau)}{T} R^{2}\left|\xi_{0}\right|^{2}-n\right) \exp \left[R^{2}\left|\xi_{0}\right|^{2} \frac{\tau(T-\tau)}{T}\right] \mathrm{d} \tau .
$$

Logo, como as duas parcelas do integrando têm o mesmo sinal,

$$
|\widehat{k}(\zeta)| \geq c_{n} n \int_{0}^{T} \exp \left[R^{2}\left|\xi_{0}\right|^{2} \frac{\tau(T-\tau)}{T}\right] \mathrm{d} \tau .
$$

Mas $\tau(T-\tau) \geq \frac{3}{16} T^{2}$ para $\tau \in\left[\frac{T}{4}, \frac{3 T}{4}\right]$. Então

$$
|\widehat{k}(\zeta)| \geq c_{n} n \frac{T}{2} \exp \left[R^{2}\left|\xi_{0}\right|^{2} \frac{3}{16} T\right]=c_{n} n \frac{T}{2} \exp \left[R^{2}\left|\xi_{0}\right|^{2} \frac{3}{16} T\right] .
$$

Agora, basta escolher $\epsilon=\min \left\{c_{n} n \frac{T}{2}, \frac{3}{16} T\right\}$. 
Procedemos agora à prova do Teorema 2.2.

Prova: Como o problema é linear, basta supor $v=0 \mathrm{em} \Omega^{*}$, e provar que $\rho \equiv 0$. Suponhamos que $\rho \not \equiv 0$ e chegaremos a um absurdo.

Como $v=0$ em $\Omega^{*} \subset W$, e $v$ é real-analítica em $W$, podemos concluir que $v$ tem suporte compacto.

Pelo Teorema de Paley-Wiener (A.5), sabemos que $\hat{v}: \mathbb{C}^{n} \rightarrow \mathbb{C}$ é inteira, e que existe $C>0$ tal que:

$$
|\hat{v}(\zeta)| \leq C \exp [C|\zeta|], \forall \zeta \in \mathbb{C}^{n} .
$$

Também pelo Teorema de Paley-Wiener, $\hat{\rho}$ é uma função inteira de ordem estrita 1 conforme mostrado no Apêndice $\mathrm{A}$, página 52. Como $\rho \not \equiv 0$, existe algum $\xi_{0} \in \mathbb{R}^{n}$ não nulo tal que $\hat{\rho}\left(\xi_{0}\right) \neq 0$. Isto é, a função de uma variável complexa $z \mapsto \hat{\rho}\left(z \xi_{0}\right)$ é inteira e não identicamente nula. Tomando $C_{1}=\left|\xi_{0}\right|^{-1}$, pelo Teorema do Mínimo Módulo (A.6), existe uma seqüência $\left(z_{j}\right) \subset \mathbb{C}, z_{j}=\imath R_{j}, R_{j}>0, R_{j} \rightarrow+\infty$ tal que

$$
\left|\hat{\rho}\left(z_{j} \xi_{0}\right)\right|>\exp \left[-C_{1}\left|z_{j}\right|\left|\xi_{0}\right|\right]=\exp \left[-C_{1} R_{j}\left|\xi_{0}\right|\right] \text {. }
$$

Usando em (2.7) a mesma seqüência $\left(z_{j}\right)$ utilizada a pouco, temos:

$$
\hat{k}\left(\imath R_{j} \xi_{0}\right) \hat{\rho}\left(\imath R_{j} \xi_{0}\right)=\hat{v}\left(\imath R_{j} \xi_{0}\right)
$$

ou seja,

$$
\hat{k}\left(\imath R_{j} \xi_{0}\right)=\frac{\hat{v}\left(\imath R_{j} \xi_{0}\right)}{\hat{\rho}\left(\imath R_{j} \xi_{0}\right)} \leq \frac{C \exp \left[C R_{j}\left|\xi_{0}\right|\right]}{\exp \left[-C_{1} R_{j}\left|\xi_{0}\right|\right]} \leq C \exp \left[\left(C+C_{1}\right) R_{j}\left|\xi_{0}\right|\right] .
$$

Mas o crescimento obtido em (2.12) é incompatível com o resultado do Lema 2.3. Logo,

$$
\rho \equiv 0
$$




\section{Capítulo 3}

\section{Extensão do resultado de Elayyan e Isakov}

Propomos fazer uma extensão do resultado de Elayyan e Isakov, Teorema 2.1, ou equivalentemente Teorema 2.2, mostrado no capítulo anterior, no sentido que a restrição sobre o posicionamento de $\Omega^{*}$ na componente conexa ilimitada de $\complement \bar{\Omega}$ é removida.

\subsection{Recapitulação importante sobre a determinação do coeficiente de condutividade da Equação do Calor}

Recordemos a restrição imposta por Elayyan e Isakov para o posicionamento dos abertos limitados de $\mathbb{R}^{n}$ envolvidos no problema. Temos dois abertos: $\Omega^{*}$, onde são feitas as medidas da temperatura, e $\Omega$, onde está o suporte da função incógnita $\rho$. Eles são tais que sendo $W$ a componente conexa ilimitada de $\complement \bar{\Omega}$ então

$$
\overline{\Omega^{*}} \subset W
$$

Como conseqüência, temos $\overline{\Omega^{*}} \bigcap \bar{\Omega}=\emptyset$.

Esquematicamente a situação está ilustrada na Figura 3.1.

Tal restrição se deve essencialmente ao seguinte fato: com ela, a função $v: \mathbb{R}^{\mathrm{n}} \rightarrow \mathbb{R}$ definida por

$$
v(x)=A \rho(x)=\int_{\Omega} k(x-y) \rho(y) \mathrm{d} y, x \in \Omega^{*},
$$

em que

$$
k(x)=-\frac{1}{4^{n+1} \pi^{n}} \int_{0}^{T} \frac{|x|^{2}}{((T-\tau) \tau)^{n / 2+1}} \exp \left[-\frac{|x|^{2} T}{4 \tau(T-\tau)}\right] \mathrm{d} \tau
$$




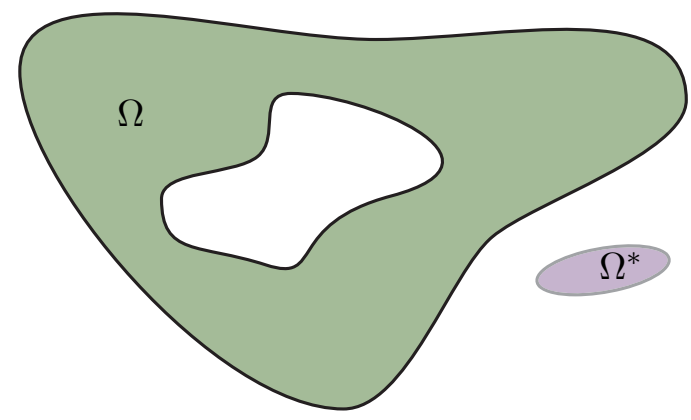

Figura 3.1: $\Omega^{*}$ está contido na componente conexa ilimitada de $\complement \Omega^{*}$.

é real analítica em $C \bar{\Omega}$. Assim, se sabemos que $v$ se anula em um conjunto qualquer que tenha um ponto de acumulação, em particular que $v$ se anula no aberto $\Omega^{*} \subset W$, então $v$ terá suporte compacto, e o Teorema de Paley-Wiener pode ser aplicado para fornecer uma estimativa para o crescimento da transformada de Fourier de $v$, como foi feito no capítulo anterior. A consequiência é a prova de unicidade para o problema inverso (2.5), sob aquela restrição topológica.

Por outro lado, se a restrição $\Omega^{*} \subset W$ for removida, a função $v$ pode não possuir suporte compacto, e o argumento não pode ser repetido.

\subsection{Proposta do problema a ser resolvido na tese}

Propomos retomar o problema da prova da unicidade para a determinação do coeficiente de condutividade $\rho$ da Equação do Calor (1.1) em sua versão linearizada (1.8), mas sem a restrição (3.1). Especificamente, consideraremos também o caso para $\Omega$ e $\Omega^{*}$ como esquematizado na Figura 3.2.

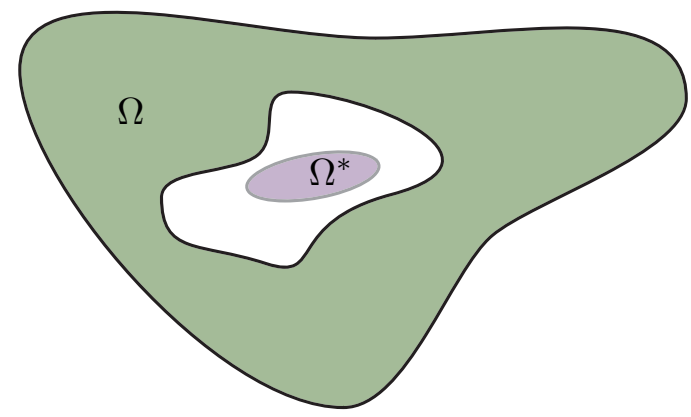

Figura 3.2: Posicionamento relativo entre $\Omega$ e $\Omega^{*}$ que será coberto pela extensão proposta do Teorema (2.2) 
Especificamente, propomos provar o teorema:

Teorema 3.1. Sejam dois abertos limitados não vazios de $\mathbb{R}^{\mathrm{n}}, n$ ímpar, $\Omega$ e $\Omega^{*}$, tais que $\bar{\Omega} \bigcap \overline{\Omega^{*}}=\emptyset$, uma função $\rho \in \mathcal{C}^{n}\left(\mathbb{R}^{\mathrm{n}}\right)$ com suporte $\operatorname{supp}(\rho) \subset \Omega$, um ponto $x_{0} \in \Omega^{*}$ e uma solução $u_{x_{0}}$ da equação

$$
\begin{cases}\frac{\partial u_{x_{0}}}{\partial t}-\Delta u_{x_{0}}=\operatorname{div}\left(\rho \vec{\nabla} \dot{u}_{x_{0}}\right), & \text { em }[0,+\infty) \times \mathbb{R}^{\mathrm{n}}, \\ u_{x_{0}}=0, & \text { em }\{0\} \times \mathbb{R}^{\mathrm{n}},\end{cases}
$$

em que $\dot{u}_{x_{0}}$, a solução fundamental da Equação do Calor, é dada por

$$
\dot{u}_{x_{0}}= \begin{cases}\frac{1}{(\sqrt{4 \pi t})^{n}} \exp \left[-\frac{\left\|x-x_{0}\right\|^{2}}{4 t}\right], & t>0, \\ 0, & t \leq 0 .\end{cases}
$$

Então, o dado sintetizado no conjunto

$$
\Gamma_{\Omega^{*}}=\left\{u_{x}(t, x) \mid x \in \Omega^{*}, a<t<b\right\},
$$

em que $0<a<b$, determina univocamente $\rho \in \mathcal{C}^{n}\left(\mathbb{R}^{\mathrm{n}}\right)$.

Observação 3.1. Como $\rho \in \mathcal{C}^{n}\left(\mathbb{R}^{\mathrm{n}}\right)$ tem suporte compacto, podemos garantir $\rho \in \mathrm{H}^{(n-1)+1}\left(\mathbb{R}^{\mathrm{n}}\right)$. Como

$$
n-1 \geq[n / 2], \quad \forall n=1,2, \ldots
$$

a Proposição 1.1 provada na página 5 garante que a solução da Equação do Calor (1.5) é contínua tanto na variável temporal como na espacial.

\subsection{Transformação do problema}

Para provar o teorema posto na seção precedente, iremos transformar a Equação (3.2) em uma Equação da Onda na forma

$$
\begin{cases}\frac{\partial^{2} \tilde{u}_{x_{0}}}{\partial \tau^{2}}(\tau, x)-\triangle \tilde{u}_{x_{0}}(\tau, x)=g_{x_{0}}(\tau, x), & \forall(\tau, x) \in[0,+\infty) \times \mathbb{R}^{\mathrm{n}}, \\ \tilde{u}_{x_{0}}(\tau, x)=0, & \forall(\tau, x) \in\{0\} \times \mathbb{R}^{\mathrm{n}}, \\ \frac{\partial \tilde{u}_{x_{0}}}{\partial \tau}(\tau, x)=0, & \forall(\tau, x) \in\{0\} \times \mathbb{R}^{\mathrm{n}},\end{cases}
$$

em que $x_{0} \in \Omega^{*}$, e $g_{x_{0}}(\tau, x)$ é uma distribuição ${ }^{1}$ a ser definida posteriormente nas variáveis $\tau$ e $x$.

\footnotetext{
${ }^{1}$ Por uma questão de clareza, escrevemos $g_{x_{0}}(\tau, x)$ para designar as variáveis em que a distribuição age.
} 


\subsubsection{Transformação da Equação do Calor em um problema hiperbólico}

Precisaremos do espaço das funções rapidamente decrescentes $\mathcal{S}\left(\mathbb{R}, \mathcal{C}_{\mathrm{c}}^{\infty}\left(\mathbb{R}^{n}\right)\right)$, definido como sendo o conjunto das funções $\phi \in \mathcal{C}^{\infty}\left(\mathbb{R}, \mathcal{C}_{\mathrm{c}}^{\infty}\left(\mathbb{R}^{n}\right)\right)$ tais que $\forall k, m \geq 0$,

$$
\left(t^{k} \frac{\mathrm{d}^{m} \phi}{\mathrm{d} t^{m}}(t)\right) \stackrel{|t| \rightarrow+\infty}{\longrightarrow} 0 \mathrm{em} \mathcal{C}_{\mathrm{c}}^{\infty}\left(\mathbb{R}^{n}\right)
$$

Observação 3.2. Podemos interpretar o espaço $\mathcal{S}\left(\mathbb{R}, \mathcal{C}_{\mathrm{c}}^{\infty}\left(\mathbb{R}^{n}\right)\right)$ fazendo a identificação de cada um de seus elementos $t \mapsto \phi(t) \in \mathcal{C}_{\mathrm{c}}^{\infty}\left(\mathbb{R}^{n}\right)$ com uma função $\tilde{\phi}$ em $\mathcal{C}^{\infty}\left(\mathbb{R} \times \mathbb{R}^{\mathrm{n}}\right)$ tal que $\tilde{\phi}(t, x)=\phi(t)(x)$, e que $\tilde{\phi}$ tem as propriedades:

1. Para todo $t \in \mathbb{R}$ fixo, $\tilde{\phi}(t, \cdot) \in \mathcal{C}_{\mathrm{c}}^{\infty}\left(\mathbb{R}^{n}\right)$.

2. Para todo $x \in \mathbb{R}^{\mathrm{n}}$ fixo, $\tilde{\phi}(\cdot, x) \in \mathcal{S}(\mathbb{R})$.

Devido à naturalidade da identificação, a seguir usaremos indistintamente $\phi$ também para denotar $\tilde{\phi}$, sendo o significado claro dentro do contexto.

Devemos fazer mais algumas definições importantes.

\section{Definição 3.1.}

$$
\begin{aligned}
& \mathcal{S}\left([0,+\infty), \mathcal{C}_{\mathrm{c}}^{\infty}\left(\mathbb{R}^{n}\right)\right)=\left\{\left.\phi\right|_{[0,+\infty)} \mid \phi \in \mathcal{S}\left(\mathbb{R}, \mathcal{C}_{\mathrm{c}}^{\infty}\left(\mathbb{R}^{n}\right)\right)\right\} \\
& \dot{\mathcal{S}}\left([0,+\infty), \mathcal{C}_{\mathrm{c}}^{\infty}\left(\mathbb{R}^{n}\right)\right)=\left\{\phi \in \mathcal{S}\left([0,+\infty), \mathcal{C}_{\mathrm{c}}^{\infty}\left(\mathbb{R}^{n}\right)\right) \mid \frac{\mathrm{d}^{m} \phi}{\mathrm{d} t^{m}}(0)=0, \forall m \in \mathbb{Z}_{+}\right\} .
\end{aligned}
$$

Os espaços duais de $\mathcal{S}\left([0,+\infty), \mathcal{C}_{\mathrm{c}}^{\infty}\left(\mathbb{R}^{n}\right)\right)$ e de $\dot{\mathcal{S}}\left([0,+\infty), \mathcal{C}_{\mathrm{c}}^{\infty}\left(\mathbb{R}^{n}\right)\right)$ são denotados por $\mathcal{S}^{\prime}\left([0,+\infty), \mathcal{D}^{\prime}\left(\mathbb{R}^{n}\right)\right)$ e por $\dot{\mathcal{S}}^{\prime}\left([0,+\infty), \mathcal{D}^{\prime}\left(\mathbb{R}^{n}\right)\right)$ respectivamente.

Para o caso em que $v \in \dot{\mathcal{S}}^{\prime}\left([0,+\infty), \mathcal{D}^{\prime}\left(\mathbb{R}^{n}\right)\right)$ tem representação por uma função, o pareamento dual entre $v$ e $\phi \in \mathcal{S}\left([0,+\infty), \mathcal{C}_{\mathrm{c}}^{\infty}\left(\mathbb{R}^{n}\right)\right)$ exprime-se por

$$
\langle v, \phi\rangle=\int_{0}^{+\infty} v(\tau) \phi(\tau) \mathrm{d} \tau .
$$

Considere a transformação

$$
\mathcal{H}: \dot{\mathcal{S}}\left([0,+\infty), \mathcal{C}_{\mathrm{c}}^{\infty}\left(\mathbb{R}^{n}\right)\right) \rightarrow \mathcal{S}\left([0,+\infty), \mathcal{C}_{\mathrm{c}}^{\infty}\left(\mathbb{R}^{n}\right)\right)
$$

definida por

$$
\mathcal{H}(\psi)(\tau)(x)=\int_{0}^{+\infty} \frac{1}{\sqrt{\pi t}} \exp \left[-\frac{\tau^{2}}{4 t}\right] \psi(t, x) \mathrm{d} t .
$$

A integral está bem definida pois para $x \in \mathbb{R}^{\mathrm{n}}$ fixo, $\psi(\cdot, x) \in \dot{\mathcal{S}}([0,+\infty])$. 
Formalmente, as derivadas de $\mathcal{H}(\psi)$ com relação a $\tau$ são somas finitas de parcelas múltiplas de termos do tipo

$$
T(m, j)(\tau)(x)=\int_{0}^{+\infty} \frac{\exp \left[-\frac{\tau^{2}}{4 t}\right]}{t^{m / 2}} \tau^{j} \psi(t, x) \mathrm{d} t,
$$

em que $m \in\{1,2, \ldots\}$ e $j \in\{0,1, \ldots\}$. O fato de $\psi \in \dot{S}\left([0,+\infty), \mathcal{C}_{\mathrm{c}}^{\infty}\left(\mathbb{R}^{n}\right)\right)$, é importante para que $T(m, j)$ esteja bem definido para $\tau \geq 0$.

Lema 3.2. Fixado $j \geq 1$ et $>0$,

$$
\sup _{\tau \in[0,+\infty)} \tau^{j} \exp \left[-\frac{\tau^{2}}{4 t}\right]=\left(\frac{2 j}{\mathrm{e}}\right)^{j / 2} t^{j / 2} .
$$

Prova: Basta derivar com relação à $\tau$ e igualar a zero para determinar o ponto de máximo.

Proposição 3.3. Para $\psi \in \dot{\mathcal{S}}\left([0,+\infty), \mathcal{C}_{\mathrm{c}}^{\infty}\left(\mathbb{R}^{n}\right)\right)$ vale

$$
\mathcal{H}(\psi) \in \mathcal{S}\left([0,+\infty), \mathcal{C}_{\mathrm{c}}^{\infty}\left(\mathbb{R}^{n}\right)\right),
$$

Prova:

1. Mostremos inicialmente que para a função $T$ definida em (3.6) vale

$$
T(m, j)(\tau)(x) \stackrel{\tau \rightarrow+\infty}{\longrightarrow} 0, \quad \forall m \geq 1, \forall j \geq 0 .
$$

a) Para $j=0$, temos que vale para o integrando:

$$
\left|\frac{\psi(t, x)}{t^{m / 2}} \exp \left[-\frac{\tau^{2}}{4 t}\right]\right| \leq\left|\frac{\psi(t, x)}{t^{m / 2}}\right|, \quad \forall t>0, \forall m=0,1, \ldots
$$

que por sua vez é integrável como função de $t$ em $] 0,+\infty), \forall m=0,1, \ldots$ Logo, pelo Teorema da Convergência Dominada, temos

$$
\begin{aligned}
\lim _{\tau \rightarrow+\infty} & \left|\int_{0}^{+\infty} \frac{\exp \left[-\frac{\tau^{2}}{4 t}\right]}{t^{m / 2}} \psi(t, x) \mathrm{d} t\right| \leq \\
\leq & \lim _{\tau \rightarrow+\infty} \int_{0}^{+\infty}\left|\frac{\psi(t, x)}{t^{m / 2}}\right| \exp \left[-\frac{\tau^{2}}{4 t}\right] \mathrm{d} t= \\
& =\int_{0}^{+\infty}\left|\frac{\psi(t, x)}{t^{m / 2}}\right| \lim _{\tau \rightarrow+\infty} \exp \left[-\frac{\tau^{2}}{4 t}\right] \mathrm{d} t=0, \quad \forall m=0,1, \ldots
\end{aligned}
$$


b) Para $j \geq 1$, usamos a majoração do lema 3.2.

$$
\left|\frac{\psi(t, x)}{t^{m / 2}}\right| \tau^{j} \exp \left[-\frac{\tau^{2}}{4 t}\right] \leq\left|\frac{2 j}{\mathrm{e}}\right|^{j / 2} t^{j / 2}\left|\frac{\psi(t, x)}{t^{m / 2}}\right|,
$$

que é integrável em $] 0,+\infty), \forall m=0,1, \ldots$, pois $\left.\psi \in \dot{\mathcal{S}}(] 0,+\infty), \mathcal{C}_{\mathrm{c}}^{\infty}\left(\mathbb{R}^{n}\right)\right)$.

Dai, aplicando o Teorema da Convergência Dominada, temos a conclusão

$$
\lim _{\tau \rightarrow+\infty} \int_{0}^{+\infty} \frac{\exp \left[-\frac{\tau^{2}}{4 t}\right]}{t^{m / 2}} \tau^{j} \psi(t, x) \mathrm{d} t=0 .
$$

2. Em (3.5) fazemos a identificação natural apontada na Observação 3.2:

$$
\psi(t)(x)=\psi(t, x),
$$

interpretando $\psi(t, \cdot)$ como uma função em $\mathcal{C}_{\mathrm{c}}^{\infty}\left(\mathbb{R}^{n}\right)$ para todo $t \in[0,+\infty)$ fixado.

Com essa identificação fica claro que para $\psi \in \dot{\mathcal{S}}\left([0,+\infty), \mathcal{C}_{\mathrm{c}}^{\infty}\left(\mathbb{R}^{n}\right)\right)$, vale que $\mathcal{H}(\psi)(\tau) \in$ $\mathcal{C}_{\mathrm{c}}^{\infty}\left(\mathbb{R}^{n}\right)$.

Lema 3.4 (A transposta de $\mathcal{H}$ ). A transposta de $\mathcal{H}$,

$$
{ }^{t} \mathcal{H}: \mathcal{S}^{\prime}\left([0,+\infty), \mathcal{D}^{\prime}\left(\mathbb{R}^{n}\right)\right) \rightarrow \dot{\mathcal{S}}^{\prime}\left([0,+\infty), \mathcal{D}^{\prime}\left(\mathbb{R}^{n}\right)\right)
$$

tem uma forma simples quando opera um elemento de $\mathcal{S}^{\prime}\left([0,+\infty), \mathcal{D}^{\prime}\left(\mathbb{R}^{n}\right)\right)$ da forma $v \otimes T$, com $v \in \mathcal{S}^{\prime}([0,+\infty))$ e $T \in \mathcal{D}^{\prime}\left(\mathbb{R}^{n}\right)$, isto é,

$$
{ }^{t} \mathcal{H}(v \otimes T)(t)=\frac{1}{\sqrt{\pi t}}\left\langle v(\tau), \exp \left[-\frac{\tau^{2}}{4 t}\right]\right\rangle_{\tau} \otimes T .
$$

Prova: Seja $\phi \in \dot{\mathcal{S}}\left([0,+\infty), \mathcal{C}_{\mathrm{c}}^{\infty}\left(\mathbb{R}^{n}\right)\right)$. Dai computamos:

$$
\begin{aligned}
{ }^{t} \mathcal{H}(v \otimes T)(\phi) & =(v \otimes T)(\mathcal{H} \phi)=T(v(\mathcal{H} \phi))= \\
& =T_{x}\left(v_{\tau}\left(\int_{0}^{+\infty} \frac{1}{\sqrt{\pi t}} \exp \left[-\frac{\tau^{2}}{4 t}\right] \phi(t, x) \mathrm{d} t\right)\right)= \\
& =\int_{0}^{+\infty} T_{x}\left(\frac{1}{\sqrt{\pi t}}\left\langle v(\tau), \exp \left[-\frac{\tau^{2}}{4 t}\right]\right\rangle_{\tau} \phi(t, x)\right) \mathrm{d} t= \\
& =\int_{0}^{+\infty} \frac{1}{\sqrt{\pi t}}\left\langle v(\tau), \exp \left[-\frac{\tau^{2}}{4 t}\right]\right\rangle_{\tau} T_{x}(\phi(t, x)) \mathrm{d} t .
\end{aligned}
$$

Obtemos então

$$
{ }^{t} \mathcal{H}(v \otimes T)(t)=\frac{1}{\sqrt{\pi t}}\left\langle v(\tau), \exp \left[-\frac{\tau^{2}}{4 t}\right]\right\rangle_{\tau} \otimes T, \forall \tau \in[0,+\infty) .
$$


Observação 3.3. Somas finitas de elementos da forma $v \otimes T$, com $v \in \mathcal{S}^{\prime}([0,+\infty))$ e $T \in$ $\mathcal{D}^{\prime}\left(\mathbb{R}^{n}\right)$ são densos em $\mathcal{S}^{\prime}\left([0,+\infty), \mathcal{D}^{\prime}\left(\mathbb{R}^{n}\right)\right)$, isto é, sendo

$$
M=\left\{\sum_{m \text { termos }} v \otimes T \mid v \in \mathcal{S}^{\prime}([0,+\infty)), T \in \mathcal{D}^{\prime}\left(\mathbb{R}^{n}\right), m \in \mathbb{N}\right\},
$$

então

$$
\bar{M}=\mathcal{S}^{\prime}\left([0,+\infty), \mathcal{D}^{\prime}\left(\mathbb{R}^{n}\right)\right) .
$$

Desejamos agora provar o seguinte teorema:

Teorema 3.5 (Conversão entre a Equação do Calor e da Onda). Se vale

$$
\operatorname{div}\left(\rho \vec{\nabla} \dot{u}_{x_{0}}\right)(t, x)={ }^{t} \mathcal{H}\left(g_{x_{0}}\right)(t, x),
$$

então os problemas (3.2) e (3.4) são relacionados por

$$
u_{x_{0}}(t, x)={ }^{t} \mathcal{H}\left(\tilde{u}_{x_{0}}\right)(t, x) .
$$

Para atingir tal objetivo, precisamos de alguns resultados intermediários.

O difeomorfismo $\left.\left.z:] 0,+\infty) \times \mathbb{R}^{\mathrm{n}} \rightarrow\right] 0,+\infty\right) \times \mathbb{R}^{\mathrm{n}}$ definido por

$$
z(t, x)=(\sqrt{t}, x)
$$

será útil.

Existe uma relação entre a transformação ${ }^{t} \mathcal{H}$ e a transformada de Laplace (ver A.6) $\mathcal{L}$ : $\mathcal{S}^{\prime}\left([0,+\infty), \mathcal{D}^{\prime}\left(\mathbb{R}^{n}\right)\right) \rightarrow \dot{\mathcal{S}}^{\prime}\left([0,+\infty), \mathcal{D}^{\prime}\left(\mathbb{R}^{n}\right)\right)$, que exploraremos agora.

Lema 3.6 (Relação entre ${ }^{t} \mathcal{H}$ e $\mathcal{L}$ ). Para $v \otimes T$, com $v \in \mathcal{S}^{\prime}([0,+\infty))$ e $T \in \mathcal{D}^{\prime}\left(\mathbb{R}^{n}\right)$, vale

$$
{ }^{t} \mathcal{H}(v \otimes T)(t)=\frac{1}{2 \sqrt{\pi t}} \mathcal{L}_{s}\left(\frac{z^{*} v \otimes T}{\sqrt{s}}\right)\left(\frac{1}{4 t}\right) .
$$

Prova: Do Lema 3.4, temos

$$
{ }^{t} \mathcal{H}(v \otimes T)(t)=\frac{1}{\sqrt{\pi t}}\left\langle v \otimes T, \exp \left[\frac{-\tau^{2}}{4 t}\right]\right\rangle_{\tau} .
$$

Usando agora o difeomorfismo $z$ definido anteriormente:

$$
\begin{aligned}
\frac{1}{\sqrt{\pi t}}\left\langle v \otimes T, \exp \left[\frac{-\tau^{2}}{4 t}\right]\right\rangle_{\tau} & =\frac{1}{\sqrt{\pi t}}\left\langle z^{*} v \otimes T, \frac{\exp \left[\frac{-s}{4 t}\right]}{2 \sqrt{s}}\right\rangle_{s}= \\
& =\frac{1}{2 \sqrt{\pi t}}\left\langle\frac{z^{*} v \otimes T}{\sqrt{s}}, \exp \left[\frac{-s}{4 t}\right]\right\rangle_{s}= \\
& =\frac{1}{2 \sqrt{\pi t}} \mathcal{L}_{s}\left(\frac{z^{*} v \otimes T}{\sqrt{s}}\right)\left(\frac{1}{4 t}\right) .
\end{aligned}
$$


Podemos expressar (3.8) usando a transformada de Laplace. A vantagem da operação é podermos nos apropriar das propriedades bem conhecidas daquela transformação. Note que as variáveis espaciais são transparentes à transformada. Pelo Lema 3.6 e usando a densidade apontada na Observação 3.3, temos

$$
\operatorname{div}\left(\rho \vec{\nabla} \dot{u}_{x_{0}}\right)(t, x)=\frac{1}{2 \sqrt{\pi t}} \mathcal{L}_{s}\left(\frac{z^{*} g_{x_{0}}(s, x)}{\sqrt{s}}\right)\left(\frac{1}{4 t}\right),
$$

ou em uma forma mais familiar, fazendo $1 /(4 t)=p$ :

$$
\operatorname{div}\left(\rho \vec{\nabla} \dot{u}_{x_{0}}\right)\left(\frac{1}{4 p}, x\right)=\frac{\sqrt{p}}{\sqrt{\pi}} \mathcal{L}_{s}\left(\frac{z^{*} g_{x_{0}}(s, x)}{\sqrt{s}}\right)(p) .
$$

Temos, então, formalmente

$$
\begin{aligned}
z^{*} g_{x_{0}}(s, x) & =\sqrt{s} \mathcal{L}_{p}^{-1}\left(\frac{\sqrt{\pi}}{\sqrt{p}} \operatorname{div}\left(\rho \vec{\nabla} \dot{u}_{x_{0}}\right)\left(\frac{1}{4 p}, x\right)\right)(s) \\
& =\lim _{m \rightarrow+\infty} \frac{-\imath \sqrt{s}}{2 \pi} \int_{\gamma-\imath m}^{\gamma+\imath m} \frac{\sqrt{\pi}}{\sqrt{p}} \operatorname{div}\left(\rho \vec{\nabla} \dot{u}_{x_{0}}\right)\left(\frac{1}{4 p}, x\right) \exp [p s] \mathrm{d} p,
\end{aligned}
$$

em que $\gamma>0$, arbitrário, é tal que todas as singularidades do integrando pertencem ao conjunto $\{\operatorname{Re} p<\gamma\}$.

Para de fato provar que (3.11) faz sentido no caso particular de nosso problema, devemos mostrar que existe $c>0$ tal que, para cada $x \in \mathbb{R}^{\mathrm{n}}$ fixo,

$$
p \mapsto \frac{\sqrt{\pi}}{\sqrt{p}} \operatorname{div}\left(\rho \vec{\nabla} \dot{u}_{x_{0}}\right)\left(\frac{1}{4 p}, x\right)
$$

é uma função holomorfa em $p$ de crescimento no máximo polinomial na região $\operatorname{Re}(p)>c$ (ver Teorema A.7). Esta condição é suficiente para que $s^{-1 / 2} g_{x_{0}}(\sqrt{s}, x) \in \mathcal{D}^{\prime}\left([0,+\infty] \times \mathbb{R}^{\mathrm{n}}\right)$ seja a transformada inversa que satisfaz (3.10).

Proposição 3.7. $p \mapsto \frac{\sqrt{\pi}}{\sqrt{p}} \operatorname{div}\left(\rho \vec{\nabla} \dot{u}_{x_{0}}\right)\left(\frac{1}{4 p}, x\right)$ é uma função holomorfa em plimitada superiormente por um polinômio em $|p|$ na região $\operatorname{Re}(p)>0$.

Prova: Na expressão de $\dot{u}_{x_{0}}$ fazemos $x=x_{0}+r \xi$, em que $r>0$ e $|\xi|=1$, para obter

$$
\vec{\nabla} \dot{u}_{x_{0}}(t, r, \xi)=\frac{-2 r}{(4 t)^{1+n / 2} \pi^{n / 2}} \exp \left[\frac{-r^{2}}{4 t}\right] \xi .
$$

Assim:

$$
\begin{aligned}
\operatorname{div}\left(\rho \vec{\nabla} \dot{u}_{x_{0}}\right)\left(\frac{1}{4 p}, r, \xi\right)= & \frac{-2 p^{1+n / 2}}{\pi^{n / 2}}\left[3 \rho\left(x_{0}, r, \xi\right)+\right. \\
& \left.r \frac{\partial \rho}{\partial r}\left(x_{0}, r, \xi\right)-2 r^{2} p \rho\left(x_{0}, r, \xi\right)\right] \exp \left[-p r^{2}\right] .
\end{aligned}
$$


Agora é fácil de se concluir o desejado.

Na fórmula acima, escrevemos $\rho\left(x_{0}, r, \xi\right)$ em vez de simplesmente $\rho(r, \xi)$ para lembrar que o sistema de coordenadas polares está centrado em $x_{0}$.

A Proposição 3.7 justifica o cálculo explícito de $g_{x_{0}}$.

$$
\begin{aligned}
z^{*} g_{x_{0}}(s, x)= & \sqrt{s} \mathcal{L}_{p}^{-1}\left[\frac{\sqrt{\pi}}{\sqrt{p}} \operatorname{div}\left(\rho \vec{\nabla} \dot{u}_{x_{0}}\right)\left(\frac{1}{4 p}, x\right)\right]= \\
= & \sqrt{s} \mathcal{L}_{p}^{-1}\left[\frac { - 2 p ^ { 1 / 2 + n / 2 } } { \pi ^ { n / 2 - 1 / 2 } } \left(3 \rho\left(x_{0}, r, \xi\right)+\right.\right. \\
& \left.\left.r \frac{\partial \rho}{\partial r}\left(x_{0}, r, \xi\right)-2 r^{2} p \rho\left(x_{0}, r, \xi\right)\right) \exp \left[-p r^{2}\right]\right](s) .
\end{aligned}
$$

Levando em conta as propriedades da transformada de Laplace (A.6.2), e que $n$ é ímpar, temos

$$
\begin{aligned}
z^{*} g_{x_{0}}(s, x)= & \frac{-2 \sqrt{s}}{\pi^{n / 2-1 / 2}}\left[3 \rho\left(x_{0}, r, \xi\right) \delta^{(1 / 2+n / 2)}\left(s-r^{2}\right)+\right. \\
& r \frac{\partial \rho}{\partial r}\left(x_{0}, r, \xi\right) \delta^{(1 / 2+n / 2)}\left(s-r^{2}\right)- \\
& \left.2 r^{2} \rho\left(x_{0}, r, \xi\right) \delta^{(3 / 2+n / 2)}\left(s-r^{2}\right)\right]
\end{aligned}
$$

em que $\delta^{(j)}$ é a $j$ - ésima derivada da distribuição $\delta$ de Dirac.

Observe que a expressão (3.11), que culminou em (3.15), permite uma extensão natural do domínio de $g_{x_{0}}$ para valores negativos da variável temporal. Explicitamente:

$$
g_{x_{0}}(s, x)=\lim _{m \rightarrow+\infty} \frac{-\imath s}{2 \pi} \int_{\gamma-\imath m}^{\gamma+\imath m} \frac{\sqrt{\pi}}{\sqrt{p}} \operatorname{div}\left(\rho \vec{\nabla} \dot{u}_{x_{0}}\right)\left(\frac{1}{4 p}, x\right) \exp \left[p s^{2}\right] \mathrm{d} p,
$$

entretanto, a estenderemos fazendo

$$
g_{x_{0}, m}(s, x)=0, \forall s<0, \forall m \in \mathbb{N} .
$$

A vantagem dessa operação ficará clara quando considerarmos a solução da Equação (3.4), como a convolução entre $g_{x_{0}}$ a sua solução fundamental.

Proposição 3.8. Existem $\tau_{0}, \tau_{1}>0$ tais que o suporte de $g_{x_{0}}$ está contido em $\left[\tau_{0}, \tau_{1}\right] \times \Omega$, $\forall x_{0} \in \Omega^{*}$.

Demonstração. Como $\bar{\Omega} \cap \overline{\Omega^{*}}=\emptyset$, vale $\operatorname{dist}\left(\Omega, \Omega^{*}\right) \doteq \sqrt{\tau_{0}}>0$.

Como $\operatorname{supp}(\rho) \subset \Omega$ e $x_{0} \in \Omega^{*}$, se $r<\sqrt{\tau_{0}}$ então $\rho\left(x_{0}, r, \xi\right)=0$ para todas as direções $\xi$. 
Além disso, como $\rho$ tem suporte compacto em $\Omega$, para $r>\operatorname{diam}\left(\Omega^{*} \bigcup \Omega\right) \doteq \sqrt{\tau_{1}}<+\infty$, temos $\rho\left(x_{0}, r, \xi\right)=0$ para todas as direções $\xi$.

Desses argumentos e mais do fato que $\operatorname{supp}(\delta)^{(j)}\left(s-r^{2}\right)=\left\{s=r^{2}\right\}, \forall j \in \mathbb{Z}_{+}$, o resultado segue imediatamente.

Uma solução de (3.4) é dada por

$$
\tilde{u}_{x_{0}}(\tau, x)=\Phi_{+} * g_{x_{0}}(\tau, x),
$$

em que $\Phi_{+}$é a solução fundamental da Equação da Onda mostrada na Fórmula (A.9) do apêndice, que tem suporte em $\tau \geq 0$.

Para comprovar que (3.16) é mesmo solução de (3.4), é necessário verificar se as condições iniciais estão satisfeitas.

Proposição 3.9. Existe um $\tau_{0}>0$ tal que a distribuição $\tilde{u}_{x_{0}}(\tau, x)$ descrita em (3.16) tem suporte contido em $\left[\tau_{0},+\infty\right) \times \mathbb{R}^{\mathrm{n}}, \forall x_{0} \in \Omega^{*}$.

Prova: Da Proposição 3.8, existem um $\tau_{0}>0$ e $\tau_{1}>0$ tais que

$$
\operatorname{supp}\left(g_{x_{0}}\right) \subset\left[\tau_{0}, \tau_{1}\right] \times \Omega \text {. }
$$

Assim, pelo Teorema dos Suportes, temos que

$$
\operatorname{supp}\left(\tilde{u}_{x_{0}}\right)=\operatorname{supp}\left(\Phi_{+} * g_{x_{0}}\right) \subset \operatorname{supp}\left(\Phi_{+}\right)+\operatorname{supp}\left(g_{x_{0}}\right)=\left[\tau_{0},+\infty\right) \times \mathbb{R}^{\mathrm{n}} .
$$

Agora estamos prontos para a prova do Teorema 3.5, enunciado na página 19.

Prova:(Do Teorema 3.5) Queremos provar que ${ }^{t} \mathcal{H} \tilde{u}_{x_{0}}$ é solução do problema parabólico (3.2). Faremos a prova em etapas.

1) Provemos, em primeiro lugar, a compatibilidade com a condição inicial de (3.2). Como elemento de $\mathcal{D}^{\prime}\left(\mathbb{R}^{n}\right)$, temos

$$
\left({ }^{t} \mathcal{H} \tilde{u}_{x_{0}}\right)(t)=\frac{1}{\sqrt{\pi t}}\left\langle\tilde{u}_{x_{0}}(\tau), \exp \left[-\frac{\tau^{2}}{4 t}\right]\right\rangle_{\tau} .
$$

Usando o resultado da Proposição 3.9 e a continuidade no espaço das funções teste, temos que $\left({ }^{t} \mathcal{H} \tilde{u}_{x_{0}}\right)(t) \stackrel{t \rightarrow 0^{+}}{\longrightarrow} 0$ em $\mathcal{D}^{\prime}\left(\mathbb{R}^{n}\right)$.

2) Provemos agora que para $\phi \in \dot{\mathcal{S}}\left([0,+\infty), \mathcal{C}_{\mathrm{c}}^{\infty}\left(\mathbb{R}^{n}\right)\right)$ vale

$$
\mathcal{H}\left(\frac{\partial \phi}{\partial t}\right)=-\frac{\partial^{2}(\mathcal{H} \phi)}{\partial \tau^{2}}
$$


De fato, uma integração por partes revela que

$$
\begin{aligned}
\mathcal{H}\left(\frac{\partial \phi}{\partial t}\right) & =\int_{0}^{+\infty} \frac{1}{\sqrt{\pi t}} \exp \left[-\frac{\tau^{2}}{4 t}\right] \frac{\partial \phi}{\partial t} \mathrm{~d} t= \\
& =-\int_{0}^{+\infty} \phi(t) \frac{1}{\sqrt{\pi t}}\left[\frac{\tau^{2}}{4 t^{2}}-\frac{1}{2 t}\right] \exp \left[-\frac{\tau^{2}}{4 t}\right] \mathrm{d} t= \\
& =-\frac{\partial^{2}(\mathcal{H} \phi)}{\partial \tau^{2}}
\end{aligned}
$$

3) Provemos que para toda solução $\tilde{u}_{x_{0}}$ vale

$$
\frac{\partial\left({ }^{t} \mathcal{H} \tilde{u}_{x_{0}}\right)}{\partial t}={ }^{t} \mathcal{H}\left(\frac{\partial^{2} \tilde{u}_{x_{0}}}{\partial \tau^{2}}\right)
$$

De fato, tomando $\phi \in \dot{\mathcal{S}}\left([0,+\infty), \mathcal{C}_{\mathrm{c}}^{\infty}\left(\mathbb{R}^{n}\right)\right)$ arbitrário, temos

$$
\begin{aligned}
\left\langle\frac{\partial^{t} \mathcal{H} \tilde{u}_{x_{0}}}{\partial t}, \phi\right\rangle & =-\left\langle{ }^{t} \mathcal{H} \tilde{u}_{x_{0}}, \frac{\partial \phi}{\partial t}\right\rangle= \\
& =-\left\langle\tilde{u}_{x_{0}}, \mathcal{H}\left(\frac{\partial \phi}{\partial t}\right)\right\rangle= \\
& =\left\langle\tilde{u}_{x_{0}}, \frac{\partial^{2} \mathcal{H} \phi}{\partial^{2} \tau}\right\rangle= \\
& =\left\langle\frac{\partial^{2} \tilde{u}_{x_{0}}}{\partial \tau^{2}},(\mathcal{H} \phi)\right\rangle= \\
& =\left\langle{ }^{t} \mathcal{H}\left(\frac{\partial^{2} \tilde{u}_{x_{0}}}{\partial \tau^{2}}\right), \phi\right\rangle .
\end{aligned}
$$

4) Como a transformação ${ }^{t} \mathcal{H}$ não envolve a variável espacial, é claro que

$$
\left\langle\triangle\left({ }^{t} \mathcal{H} \tilde{u}_{x_{0}}\right), \phi\right\rangle=\left\langle\triangle \tilde{u}_{x_{0}}, \mathcal{H} \phi\right\rangle
$$

5) Dos itens precedentes 3 e 4 e da Hipótese (3.8), vem que para qualquer $\phi \in \dot{\mathcal{S}}\left([0,+\infty), \mathcal{C}_{\mathrm{c}}^{\infty}\left(\mathbb{R}^{n}\right)\right)$ temos:

$$
\begin{aligned}
& \left\langle\left[\frac{\partial}{\partial t}-\triangle\right]{ }^{t} \mathcal{H}\left(\tilde{u}_{x_{0}}\right), \phi\right\rangle=\left\langle\left[\frac{\partial^{2}}{\partial \tau^{2}}-\triangle\right] \tilde{u}_{x_{0}}, \mathcal{H} \phi\right\rangle= \\
& =\langle{ }^{t} \mathcal{H} \underbrace{\left[\frac{\partial^{2}}{\partial \tau^{2}}-\triangle\right] \tilde{u}_{x_{0}}}_{g_{x_{0}}}, \phi\rangle=\left\langle\operatorname{div}\left(\rho \vec{\nabla} \dot{u}_{x_{0}}\right), \phi\right\rangle .
\end{aligned}
$$




\subsubsection{Solução do problema hiperbólico}

Começamos com um lema básico.

Lema 3.10 (Continuidade na convolução). Se $g_{m} \stackrel{m \rightarrow+\infty}{\longrightarrow} g$ em $\mathcal{D}^{\prime}([0,+\infty) \times \Omega)$ e $f \in$ $\mathcal{D}^{\prime}\left([0,+\infty) \times \mathbb{R}^{\mathrm{n}}\right)$, com $\Omega \subset \mathbb{R}^{\mathrm{n}}$ limitado, então

$$
\lim _{m \rightarrow+\infty} f * g_{m}=f *\left(\lim _{m \rightarrow+\infty} g_{m}\right) .
$$

Prova: Tome $\phi \in \mathcal{C}_{\mathrm{c}}^{\infty}\left([0,+\infty) \times \mathbb{R}^{\mathrm{n}}\right)$ função teste.

$$
\begin{aligned}
\lim _{m \rightarrow+\infty}\left(f * g_{m}\right) * \phi & =\lim _{m \rightarrow+\infty} f *\left(g_{m} * \phi\right)= \\
& =f *(g * \phi)= \\
& =(f * g) * \phi= \\
& =f *\left(\lim _{m \rightarrow+\infty} g_{m}\right) * \phi .
\end{aligned}
$$

Logo, como $\phi$ é arbitrária, temos

$$
\lim _{m \rightarrow+\infty} f * g_{m}=f *\left(\lim _{m \rightarrow+\infty} g_{m}\right) .
$$

Para o caso $n=1$, podemos obter uma fórmula simples para $\tilde{u}_{x_{0}}$.

Sendo $\mathrm{H}$ a função de Heaviside, a solução fundamental da Equação da Onda para $n=1$ é dada por

$$
\Phi_{+}(\tau, x)=\frac{1}{2} \mathrm{H}(\tau-|x|) .
$$

De (3.11) e (3.16), usando o Lema 3.10, obtemos a fórmula para a solução do problema hiperbólico (3.4) para $n=1$ :

$$
\begin{aligned}
\tilde{u}_{x_{0}}(\tau, x)= & \Phi_{+} *_{t, \xi} \lim _{m \rightarrow+\infty}\left[\frac{-\imath t}{2 \pi} \times\right. \\
& \left.\int_{\gamma-\imath m}^{\gamma+\imath m} \frac{\sqrt{\pi}}{\sqrt{p}} \operatorname{div}\left(\rho \vec{\nabla} \dot{u}_{x_{0}}\right)\left(\frac{1}{4 p}, \xi\right) \exp \left[p t^{2}\right] \mathrm{d} p\right](\tau, x)= \\
= & \lim _{m \rightarrow+\infty}\left[\Phi_{+} *_{t, \xi} \frac{-\imath t}{2 \pi} \times\right. \\
& \left.\int_{\gamma-\imath m}^{\gamma+\imath m} \frac{\sqrt{\pi}}{\sqrt{p}} \operatorname{div}\left(\rho \vec{\nabla} \dot{u}_{x_{0}}\right)\left(\frac{1}{4 p}, \xi\right) \exp \left[p t^{2}\right] \mathrm{d} p\right](\tau, x)= \\
= & \lim _{m \rightarrow+\infty}\left[\frac{1}{2} \int_{0}^{\tau} \int_{-t}^{t} \frac{-\imath(\tau-t)}{2 \pi} \times\right. \\
& \left.\int_{\gamma-\imath m}^{\gamma+\imath m} \frac{\sqrt{\pi}}{\sqrt{p}} \operatorname{div}\left(\rho \vec{\nabla} \dot{u}_{x_{0}}\right)\left(\frac{1}{4 p}, x-\xi\right) \exp \left[p(\tau-t)^{2}\right] \mathrm{d} p \mathrm{~d} \xi \mathrm{d} t\right] .
\end{aligned}
$$


Fazendo a mudança de variáveis $y=x-\xi$, e na seqüência $z=\tau-t$, obtemos

$$
\begin{aligned}
\tilde{u}_{x_{0}}(\tau, x)= & \lim _{m \rightarrow+\infty} \frac{1}{2} \int_{0}^{\tau} \int_{x-\tau+z}^{x+\tau-z} \frac{-\imath z}{2 \pi} \times \\
& \int_{\gamma-\imath m}^{\gamma+\imath m} \frac{\sqrt{\pi}}{\sqrt{p}} \operatorname{div}\left(\rho \vec{\nabla} \dot{u}_{x_{0}}\right)\left(\frac{1}{4 p}, y\right) \exp \left[p z^{2}\right] \mathrm{d} p \mathrm{~d} y \mathrm{~d} z= \\
= & \lim _{m \rightarrow+\infty} \frac{1}{2} \int_{0}^{\tau} \int_{\gamma-\imath m}^{\gamma+\imath m} \frac{-\imath z}{2 \sqrt{\pi} \sqrt{p}} \exp \left[p z^{2}\right] \times \\
& \int_{x-\tau+z}^{x+\tau-z} \operatorname{div}\left(\rho \vec{\nabla} \dot{u}_{x_{0}}\right)\left(\frac{1}{4 p}, y\right) \mathrm{d} y \mathrm{~d} p \mathrm{~d} z .
\end{aligned}
$$

Fazendo a integração por partes da integral interna, obtemos

$$
\begin{aligned}
\tilde{u}_{x_{0}}(\tau, x)=\lim _{m \rightarrow+\infty} \frac{1}{2}\left[\int_{0}^{\tau} \int_{\gamma-\imath m}^{\gamma+\imath m} \frac{-\imath z}{2 \sqrt{\pi} \sqrt{p}} \exp \left[p z^{2}\right] \times\right. \\
\left(\rho \vec{\nabla} \dot{u}_{x_{0}}\right)\left(\frac{1}{4 p}, x+\tau-z\right) \mathrm{d} p \mathrm{~d} z- \\
\int_{0}^{\tau} \int_{\gamma-\imath m}^{\gamma+\imath m} \frac{-\imath z}{2 \sqrt{\pi} \sqrt{p}} \exp \left[p z^{2}\right] \times \\
\left.\quad\left(\rho \vec{\nabla} \dot{u}_{x_{0}}\right)\left(\frac{1}{4 p}, x-\tau+z\right) \mathrm{d} p \mathrm{~d} z\right] .
\end{aligned}
$$

Após substituir $\vec{\nabla} \dot{u}_{x_{0}}$ na fórmula acima, fazemos $x_{0}=x$ em concordância com o dado sintetizado em $\Gamma_{\Omega^{*}}$ (3.3). O resultado é

$$
\begin{aligned}
& \tilde{u}_{x}(\tau, x)=\lim _{m \rightarrow+\infty} {\left[\int_{0}^{\tau} z \rho(x+\tau-z)(z-\tau) \times\right.} \\
& \frac{-\imath}{2 \pi} \int_{\gamma-\imath m}^{\gamma+\imath m} p \exp \left[p\left(z^{2}-(\tau-z)^{2}\right)\right] \mathrm{d} p \mathrm{~d} z- \\
& \int_{0}^{\tau} z \rho(x-\tau+z)(\tau-z) \times \\
&\left.\frac{-\imath}{2 \pi} \int_{\gamma-\imath m}^{\gamma+\imath m} p \exp \left[p\left(z^{2}-(\tau-z)^{2}\right)\right] \mathrm{d} p \mathrm{~d} z\right]
\end{aligned}
$$

Fazendo a substituição

$$
z=\frac{s+\tau^{2}}{2 \tau}
$$

temos

$$
\begin{array}{r}
\tilde{u}_{x_{0}}(\tau, x)=\lim _{m \rightarrow+\infty}[ \\
\int_{-\tau^{2}}^{\tau^{2}} \phi_{1}(\tau, x, s) \frac{-\imath}{2 \pi} \int_{\gamma-\imath m}^{\gamma+\imath m} p \exp [p s] \mathrm{d} p \mathrm{~d} s+ \\
\left.\int_{-\tau^{2}}^{\tau^{2}} \phi_{2}(\tau, x, s) \frac{-\imath}{2 \pi} \int_{\gamma-\imath m}^{\gamma+\imath m} p \exp [p s] \mathrm{d} p \mathrm{~d} s\right],
\end{array}
$$


em que

$$
\begin{aligned}
& \phi_{1}(\tau, x, s)=\frac{1}{2 \tau}\left(\frac{s+\tau^{2}}{2 \tau}\right) \rho\left(x+\tau-\frac{s+\tau^{2}}{2 \tau}\right)\left(\frac{s+\tau^{2}}{2 \tau}-\tau\right) \\
& \phi_{2}(\tau, x, s)=\frac{1}{2 \tau}\left(\frac{s+\tau^{2}}{2 \tau}\right) \rho\left(x-\tau+\frac{s+\tau^{2}}{2 \tau}\right)\left(\frac{s+\tau^{2}}{2 \tau}-\tau\right)
\end{aligned}
$$

Precisaremos de alguns resultados para tratarmos o limite em (3.17).

Lema 3.11 (Riemann-Lebesgue). Seja $f$ uma função integrável no intervalo $[a, b]$. Então

$$
\lim _{m \rightarrow+\infty} \int_{a}^{b} f(t) \operatorname{sen}(t m) \mathrm{d} t=0 .
$$

Prova: Faremos a aproximação de $f$ por funções simples. Para o caso em que $f$ é uma constante $c$, a prova do lema é elementar:

$$
\int_{a}^{b} c \operatorname{sen}(t m) \mathrm{d} t=c \frac{\cos (a m)-\cos (b m)}{m} .
$$

Dai, para $m \rightarrow+\infty$, temos que

$$
\lim _{m \rightarrow+\infty} \int_{a}^{b} c \operatorname{sen}(t m) \mathrm{d} t=0 .
$$

Como $f$ é integrável, existe uma função simples $\tilde{f}$ tal que para dado $\epsilon>0$ arbitrário, vale

$$
\left|\int_{a}^{b} f(t) \operatorname{sen}(t m) \mathrm{d} t-\int_{a}^{b} \tilde{f}(t) \operatorname{sen}(t m) \mathrm{d} t\right|<\epsilon .
$$

Dai temos a prova do lema.

Lema 3.12. Para $a>0$, vale

$$
\lim _{m \rightarrow+\infty} \int_{a}^{+\infty} \frac{\operatorname{sen}(m z)}{z} \mathrm{~d} z=0 .
$$

Prova: Para todo $\epsilon>0$ arbitrário, podemos escolher $a_{\epsilon}>a$ tal que para qualquer $m \geq 1$ valha

$$
\int_{a_{\epsilon}}^{+\infty} \frac{\operatorname{sen}(m z)}{z} \mathrm{~d} z<\epsilon
$$

pois

$$
\int_{a_{\epsilon}}^{+\infty} \frac{\operatorname{sen}(m z)}{z} \mathrm{~d} z=\int_{m a_{\epsilon}}^{+\infty} \frac{\operatorname{sen}(\xi)}{\xi} \mathrm{d} \xi
$$

que é Riemann-integrável. 
Logo,

$$
\int_{a}^{+\infty} \frac{\operatorname{sen}(m z)}{z} \mathrm{~d} z \leq \int_{a}^{a_{\epsilon}} \frac{\operatorname{sen}(m z)}{z} \mathrm{~d} z+\epsilon .
$$

Como em $\left[a, a_{\epsilon}\right]$ a função $z \mapsto \frac{1}{z}$ é integrável, pelo Lema 3.11 temos

$$
\lim _{m \rightarrow+\infty} \int_{a}^{+\infty} \frac{\operatorname{sen}(m z)}{z} \mathrm{~d} z \leq \epsilon .
$$

Pelo fato de $\epsilon>0$ ser arbitrário, temos a prova do lema.

Do resultado do lema3.12, sabendo que

$$
\lim _{m \rightarrow+\infty} \int_{-\infty}^{+\infty} \frac{\operatorname{sen}(m z)}{z} \mathrm{~d} z=\pi
$$

temos os seguintes fatos:

1. Para $a, b>0$ vale

$$
\lim _{m \rightarrow+\infty} \int_{-b}^{a} \frac{\operatorname{sen}(m z)}{z} \mathrm{~d} z=\pi
$$

e

2. Para $a>0$ vale

$$
\lim _{m \rightarrow+\infty} \int_{0}^{a} \frac{\operatorname{sen}(m z)}{z} \mathrm{~d} z=\frac{\pi}{2}
$$

A seguir, usamos a função característica de um conjunto $A$ definida por

$$
\chi_{A}(x)= \begin{cases}1, & \text { if } x \in A, \\ 0, & \text { if } x \notin A .\end{cases}
$$

Lema 3.13. Seja a função $h_{m, \gamma}: \mathbb{R} \rightarrow \mathbb{C}$ definida por

$$
h_{m, \gamma}(z)=\frac{-\imath}{2 \pi} \int_{\gamma-\imath m}^{\gamma+\imath m} \exp [p z] \mathrm{d} p=\frac{1}{\pi} \frac{\operatorname{sen}(m z)}{z} \exp [\gamma z],
$$

em que $\gamma \in \mathbb{R}$. Então para $a, b>0$ vale

$$
\chi_{[-b, a]} h_{m, \gamma} \stackrel{m \rightarrow+\infty}{\longrightarrow} \delta, \forall \gamma \in \mathbb{R}
$$

no espaço das distribuições de ordem menor ou igual a 1.

Prova: Devemos provar que para toda $\phi \in \mathcal{C}_{c}^{1}(\mathbb{R})$ vale

$$
\lim _{m \rightarrow+\infty}\left(\chi_{[-b, a]} h_{m, \gamma}\right)(\phi)=\phi(0), \forall \gamma \in \mathbb{R} .
$$


Provaremos que

$$
\lim _{m \rightarrow+\infty} \int_{-b}^{a} \frac{1}{\pi} \exp [\gamma z] \frac{\operatorname{sen}(m z)}{z} \phi(z) \mathrm{d} z=\phi(0)
$$

Devido à (3.18), podemos escrever

$$
\phi(0)=\lim _{m \rightarrow+\infty} \frac{1}{\pi} \int_{-b}^{a} \phi(0) \exp [\gamma 0] \frac{\operatorname{sen}(m z)}{z} \mathrm{~d} z .
$$

Logo,

$$
\begin{aligned}
\lim _{m \rightarrow+\infty} \quad & \int_{-b}^{a} \frac{1}{\pi} \exp [\gamma z] \frac{\operatorname{sen}(m z)}{z} \phi(z) \mathrm{d} z-\phi(0)= \\
& \lim _{m \rightarrow+\infty} \frac{1}{\pi} \int_{-b}^{a} \frac{\phi(z) \exp [\gamma z]-\phi(0) \exp [\gamma 0]}{z} \operatorname{sen}(m z) \mathrm{d} z .
\end{aligned}
$$

Como $\phi \in \mathcal{C}_{c}^{1}(\mathbb{R})$, pelo Lema 3.11, temos imediatamente que

$$
\lim _{m \rightarrow+\infty} \int_{-b}^{a} \frac{1}{\pi} \exp [\gamma z] \frac{\operatorname{sen}(m z)}{z} \phi(z) \mathrm{d} z-\phi(0)=0 .
$$

Corolário 3.14. Com pequenas alterações na prova do Lema 3.13, temos que para qualquer função teste $\phi \in \mathcal{C}_{c}^{1}(\mathbb{R}), \forall a, b, \gamma \in \mathbb{R}, 0 \leq b<a$ vale

$$
\lim _{m \rightarrow+\infty}\left(\chi_{[b, a]} h_{m, \gamma}\right)(\phi)= \begin{cases}\frac{\phi(0)}{2}, & b=0 \\ 0, & b>0 .\end{cases}
$$

Lema 3.15. Dados $a, b>0, q \in\{1,2, \ldots\}$ e $\phi \in \mathcal{C}^{q}(\mathbb{R})$ tais que $\phi^{(j)}(-b)=\phi^{(j)}(a)=0$, $j \in\{0,1, \ldots(q-1)\}$, então

$$
\lim _{m \rightarrow+\infty} \int_{-b}^{+a} h_{m, \gamma}^{(q)}(z) \phi(z) \mathrm{d} z=(-1)^{q} \phi^{(q)}(0), \forall \gamma \in \mathbb{R} .
$$

Prova: Pela Fórmula de Leibniz para distribuições temos

$$
\left(\chi_{[-b, a]} h_{m, \gamma}\right)^{(q)}=\chi_{[-b, a]} h_{m, \gamma}^{(q)}+\sum_{k=1}^{q}\left(\begin{array}{l}
q \\
k
\end{array}\right) h_{m, \gamma}^{(q-k)}\left(\delta_{-b}-\delta_{a}\right)^{(k-1)} .
$$

Isto é,

$$
\chi_{[-b, a]} h_{m, \gamma}^{(q)}=\left(\chi_{[-b, a]} h_{m, \gamma}\right)^{(q)}-\sum_{k=1}^{q}\left(\begin{array}{l}
q \\
k
\end{array}\right) h_{m, \gamma}^{(q-k)}\left(\delta_{-b}-\delta_{a}\right)^{(k-1)} .
$$


Por outro lado,

$$
\begin{aligned}
\chi_{[-b, a]} h_{m, \gamma} & \stackrel{\mathcal{D}^{\prime}}{\longrightarrow} \delta, \\
\left(\chi_{[-b, a]} h_{m, \gamma}\right)^{(q)} & \stackrel{\mathcal{D}^{\prime}}{\longrightarrow} \delta^{(q)}, \forall \gamma \in \mathbb{R}
\end{aligned}
$$

De (3.21) e (3.22), vem que $\forall \gamma \in \mathbb{R}$ :

$$
\begin{aligned}
\lim _{m \rightarrow+\infty} \chi_{[-b, a]} h_{m, \gamma}^{(q)}(\phi) & =\delta^{(q)}(\phi)- \\
& \lim _{m \rightarrow+\infty} \sum_{k=1}^{q}\left(\begin{array}{l}
q \\
k
\end{array}\right)\left(\delta_{-b}-\delta_{a}\right)^{(k-1)}\left(h_{m, \gamma}^{(q-k)} \phi\right) .
\end{aligned}
$$

Mas lembrando que por hipótese $\phi^{(j)}(-b)=\phi^{(j)}(a)=0$, para $j \in\{0,1, \ldots,(q-1)\}$, temos:

$$
\lim _{m \rightarrow+\infty} \sum_{k=1}^{q}\left(\begin{array}{l}
q \\
k
\end{array}\right)\left(\delta_{-b}-\delta_{a}\right)^{(k-1)}\left(h_{m, \gamma}^{(q-k)} \phi\right)=0,
$$

o que conclui a prova.

Com pequenas alterações na prova do Lema 3.15, obtemos um resultado que será útil mais tarde.

Corolário 3.16. Dados $b \geq 0, a>b, q \in\{1,2, \ldots\}$ e $\phi \in \mathcal{C}^{q}(\mathbb{R})$ tais que $\phi^{(j)}(a)=\phi^{(j)}(b)=$ $0, j \in\{0,1, \ldots(q-1)\}$ então para qualquer $\gamma \in \mathbb{R}$,

$$
\lim _{m \rightarrow+\infty} \int_{b}^{a} h_{m, \gamma}^{(q)}(z) \phi(z) \mathrm{d} z= \begin{cases}\frac{1}{2}(-1)^{q} \phi^{(q)}(0), & b=0 \\ 0, & b>0 .\end{cases}
$$

Aplicando em (3.17) o Lema 3.15, notando que $\phi_{1}$ e $\phi_{2}$ têm zeros em $-\tau^{2}$ e em $\tau^{2}$, temos para $n=1$ a solução

$$
\tilde{u}_{x}(\tau, x)=\frac{1}{16}\left[\rho^{\prime}\left(x-\frac{\tau}{2}\right)-\rho^{\prime}\left(x+\frac{\tau}{2}\right)\right], \forall \tau>0, \forall x \in \Omega^{*} .
$$

Lema 3.17. Seja $F: \mathbb{R} \rightarrow \mathbb{R}$ uma função compactamente suportada de classe $\mathcal{C}^{q}$, tal que $0 \notin \operatorname{supp}(F)$, e seja

$$
A(z, \tau)=F\left(\frac{\tau^{2}-z}{\tau}\right) \frac{P(z, \tau)}{\tau^{s}}
$$

em que $P$ é uma função polinomial, e $s \in\{0,1, \ldots\}$.

Existe $\epsilon>0$ tal que $0<\tau<\epsilon, z \in\left[-\tau^{2}, \tau^{2}\right] \Rightarrow A(z, \tau)=0$. 
Demonstração. Para $0 \leq z \leq \tau^{2}$,

$$
\frac{\tau^{2}-z}{\tau} \leq \tau
$$

Para $-\tau^{2} \leq z<0$,

$$
\frac{\tau^{2}-z}{\tau}<2 \tau
$$

O resultado segue, já que $0 \notin \operatorname{supp}(F)$.

Lema 3.18.

$$
B(\xi)=\frac{\partial^{l} A}{\partial z^{l}}(\xi, \sqrt{-\xi}), \xi \leq 0 .
$$

As derivadas de B são da forma

$$
\frac{\mathrm{d}^{j} B}{\mathrm{~d} \xi^{j}}=\sum_{i=0}^{j} K_{i} F^{(i+l)}(2 \sqrt{-\xi}) \frac{\tilde{P}_{i}(\sqrt{-\xi})}{\sqrt{-\xi}^{k_{i}}}, \xi \leq 0,
$$

em que $K_{i} \in \mathbb{Z}, k_{i} \in \mathbb{Z}_{+}$são constantes, e $\tilde{P}_{i}$ são funções polinomiais.

Lema 3.19. Seja $M>0$. A convergência

$$
\left(\chi_{\left[-\tau^{2}, \tau^{2}\right]} h_{m, \gamma}\right)(A(\cdot, \tau)) \stackrel{m \rightarrow+\infty}{\longrightarrow} A(0, \tau)
$$

é uniforme em $0<\tau<M$.

Demonstração. A prova segue imitando a do Lema 3.13, levando em conta as seguintes observações:

1) Do Lema 3.17, existe $\epsilon>0$ tal que $0<\tau<\epsilon$ e $z \in\left[-\tau^{2}, \tau^{2}\right]$ implica em $A(z, \tau)=0$. Isso significa que

2) a convergência

$$
\frac{1}{\pi} \int_{-\tau^{2}}^{\tau^{2}} A(0, \tau) \frac{\operatorname{sen}(m z)}{z} \mathrm{~d} z \stackrel{m \rightarrow+\infty}{\longrightarrow} A(0, \tau)
$$

é uniforme para $0<\tau<M$.

Corolário 3.20. Sob as mesmas condições do lema precedente, a convergência

$$
\left(\chi_{\left[-\tau^{2}, \tau^{2}\right]} h_{m, \gamma}\right)^{(q)}(A(\cdot, \tau)) \stackrel{m \rightarrow+\infty}{\longrightarrow}(-1)^{q} A(0, \tau)
$$

é uniforme em $0<\tau<M$, pois $A \in \mathcal{C}^{q}$. 
Lema 3.21. Seja $q \in\{1,2, \ldots\}$. Então, se definirmos para $m \in\{1,2, \ldots\}$ e $\gamma \in \mathbb{R}$ :

$$
d_{m, \gamma, q}(\tau)=\int_{-\tau^{2}}^{\tau^{2}} h_{m, \gamma}^{(q)}(z) A(z, \tau) \mathrm{d} z, \tau>0
$$

temos

$$
\lim _{m \rightarrow+\infty} d_{m, \gamma, q}(\tau)=(-1)^{q}\left(\mathrm{D}_{1}^{q} A\right)(0, \tau), \text { em } \mathcal{D}^{\prime}([0,+\infty)) .
$$

Demonstração. 1) De (3.21), e como $A(z, \tau)$ é nula perto de $z=\tau^{2}$, temos

$$
\begin{aligned}
d_{m, \gamma, q}(\tau)= & \int_{-\tau^{2}}^{\tau^{2}} h_{m, \gamma}^{(q)}(z) A(z, \tau) \mathrm{d} z= \\
= & \left(\chi_{\left[-\tau^{2}, \tau^{2}\right]} h_{m, \gamma}\right)^{(q)}(A(\cdot, \tau))- \\
& \left.\sum_{k=1}^{q}(-1)^{k-1}\left(\begin{array}{l}
q \\
k
\end{array}\right) \frac{\mathrm{d}^{k-1}}{\mathrm{~d} z^{k-1}}\left(h_{m, \gamma}^{(q-k)}(z) A(z, \tau)\right)\right|_{z=-\tau^{2}} .
\end{aligned}
$$

2) Agora aplicamos $d_{m, \gamma, q}$ a uma função teste $\phi \in \mathcal{C}_{c}^{\infty}([0,+\infty))$. Obtemos

$$
\begin{aligned}
d_{m, \gamma, q}(\phi)= & \int_{0}^{+\infty}\left(\chi_{\left[-\tau^{2}, \tau^{2}\right]} h_{m, \gamma}\right)^{(q)}(A(\cdot, \tau)) \phi(\tau) \mathrm{d} \tau- \\
& \left.\int_{0}^{+\infty} \sum_{k=1}^{q}\left(\begin{array}{l}
q \\
k
\end{array}\right)(-1)^{k-1} \frac{\mathrm{d}^{k-1}}{\mathrm{~d} z^{k-1}}\left(h_{m, \gamma}^{(q-k)}(z) A(z, \tau)\right)\right|_{z=-\tau^{2}} \phi(\tau) \mathrm{d} \tau .
\end{aligned}
$$

Pela regra de Leibniz, temos

$$
\frac{\mathrm{d}^{k-1}}{\mathrm{~d} z^{k-1}}\left(h_{m, \gamma}^{(q-k)}\left(-\tau^{2}\right) A\left(-\tau^{2}, \tau\right)\right)=\sum_{l=0}^{k-1}\left(\begin{array}{c}
k-1 \\
l
\end{array}\right) h_{m, \gamma}^{(q-k+l)}\left(-\tau^{2}\right) \frac{\mathrm{d}^{k-l-1} A}{\mathrm{~d} z^{k-l-1}}\left(-\tau^{2}, \tau\right) .
$$

Portanto,

$$
\begin{aligned}
d_{m, \gamma, q}(\phi)= & \int_{0}^{+\infty}\left(\chi_{\left[-\tau^{2}, \tau^{2}\right]} h_{m, \gamma}\right)^{(q)}(A(\cdot, \tau)) \phi(\tau) \mathrm{d} \tau- \\
& \sum_{k=1}^{q}\left(\begin{array}{l}
q \\
k
\end{array}\right) \sum_{l=0}^{k-1}\left(\begin{array}{c}
k-1 \\
l
\end{array}\right)(-1)^{k-1} \times \\
& \int_{0}^{+\infty} h_{m, \gamma}^{(q-k+l)}\left(-\tau^{2}\right) \frac{\mathrm{d}^{k-l-1} A}{\mathrm{~d} z^{k-l-1}}\left(-\tau^{2}, \tau\right) \phi(\tau) \mathrm{d} \tau .
\end{aligned}
$$

Fazemos agora a mudança de variáveis $\xi=-\tau^{2}$.

$$
\begin{aligned}
d_{m, \gamma, q}(\phi)= & \int_{0}^{+\infty}\left(\chi_{\left[-\tau^{2}, \tau^{2}\right]} h_{m, \gamma}\right)^{(q)}(A(\cdot, \tau)) \phi(\tau) \mathrm{d} \tau- \\
& \sum_{k=1}^{q}\left(\begin{array}{c}
q \\
k
\end{array}\right) \sum_{l=0}^{k-1}\left(\begin{array}{c}
k-1 \\
l
\end{array}\right)(-1)^{k-1} \int_{-\infty}^{0} h_{m, \gamma}^{(q-k+l)}(\xi) \times \\
& \frac{\mathrm{d}^{k-l-1} A}{\mathrm{~d} z^{(k-l-1)}}(\xi, \sqrt{-\xi}) \phi(\sqrt{-\xi}) \frac{1}{2 \sqrt{-\xi}} \mathrm{d} \xi
\end{aligned}
$$


Agora, o limite do segundo termo para $m \rightarrow+\infty$ é zero, pois $\phi$ tem suporte compacto, e porque $\xi \mapsto A(\xi, \sqrt{-\xi})$ é nula em uma vizinhança de $\xi=0$ pelo Lema 3.18.

3) Pelo Corolário 3.20, a convergência

$$
\left(\chi_{\left[-\tau^{2}, \tau^{2}\right]} h_{m, \gamma}\right)^{(q)}(A(\cdot, \tau)) \stackrel{m \rightarrow+\infty}{\longrightarrow}(-1)^{q} D_{1}^{q} A(0, \tau)
$$

é uniforme para $0<\tau<M$.

Portanto, temos

$$
\lim _{m \rightarrow+\infty} d_{m, \gamma, q}(\phi)=\int_{0}^{+\infty}(-1)^{q} \mathrm{D}_{1}^{q}(A(0, \tau)) \phi(\tau) \mathrm{d} \tau, \forall \gamma \in \mathbb{R} .
$$

Concluímos que $\forall \gamma \in \mathbb{R}, \lim _{m \rightarrow+\infty} d_{m, \gamma, q}$ se identifica com a função contínua $d_{q}(\tau)=$ $(-1)^{q} \mathrm{D}_{1}^{q} A(0, \tau)$, em $\mathcal{D}^{\prime}([0,+\infty))$.

Para o caso $n=3$, também partimos de

$$
\tilde{u}_{x_{0}}(x, \tau)=\Phi_{+} * g_{x_{0}},
$$

em que $\Phi_{+}$é a solução fundamental mostrada em (A.9).

Para maior conveniência, destacamos aqui a distribuição $\Phi_{+}$.

$$
\Phi_{+}(\psi)=\int_{0}^{+\infty} \frac{1}{4 \pi} t \int_{|\xi|=1} \psi(t, t \xi) \mathrm{d} \sigma_{\xi} \mathrm{d} t, \forall \psi \in \mathcal{C}_{c}^{\infty}\left(\mathbb{R}_{+} \times \mathbb{R}^{3}\right) .
$$

Observamos que a convolução entre $\Phi_{+}$e uma teste $\psi \in \mathcal{C}_{c}^{\infty}\left(\mathbb{R}_{+} \times \mathbb{R}^{3}\right)$ é dada por

$$
\Phi_{+} * \psi(\tau, x)=\Phi_{+}(\psi(\tau-\cdot, x-\cdot))=\int_{0}^{+\infty} \frac{1}{4 \pi} t \int_{|\xi|=1} \psi(\tau-t, x-t \xi) \mathrm{d} \sigma_{\xi} \mathrm{d} t .
$$

De (3.14), temos

$$
\begin{aligned}
g_{x_{0}, \gamma}(s, x)= & \lim _{m \rightarrow+\infty} \frac{s(-\imath)}{2 \pi} \int_{\gamma-\imath m}^{\gamma+\imath m} \frac{-2 p^{2}}{\pi}\left(3 \rho\left(x_{0}, r, \xi\right)+\right. \\
& \left.r \frac{\partial \rho}{\partial r}\left(x_{0}, r, \xi\right)-2 r^{2} p \rho\left(x_{0}, r, \xi\right)\right) \times \\
& \exp \left[-p r^{2}\right] \exp \left[s^{2} p\right] \mathrm{d} p, s \geq 0, x \in \mathbb{R}^{3} .
\end{aligned}
$$

em que $\gamma \in \mathbb{R}$ é arbitrário. Como discutido anteriormente, estenderemos $g_{x_{0}}$ da seguinte forma: $g_{x_{0}, \gamma}(s, x)=0$ para $s<0$. 
Por conveniência, façamos

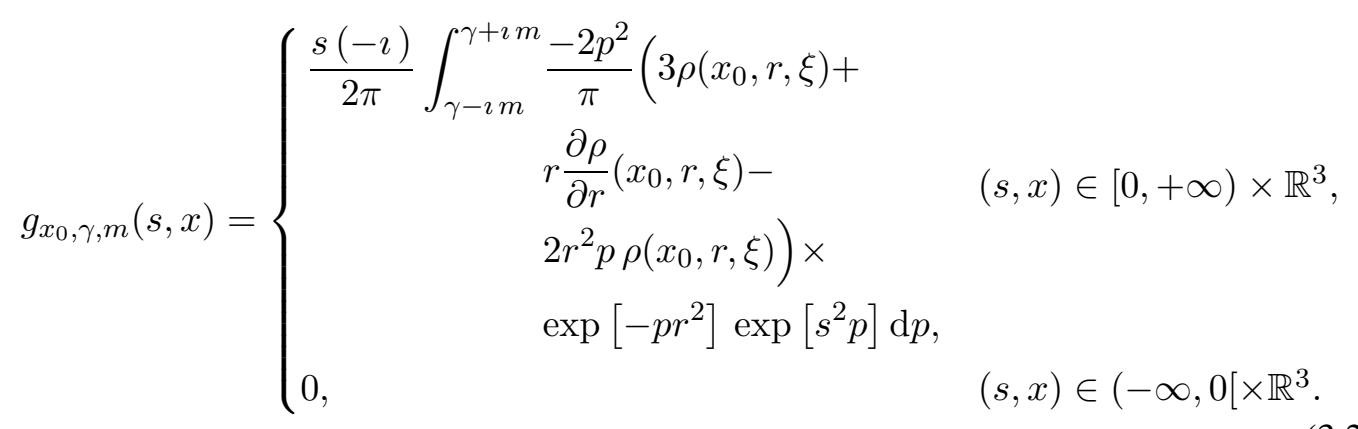

Escrevamos

$$
g_{x_{0}, \gamma}(s, x)=\lim _{m \rightarrow+\infty} g_{x_{0}, m, \gamma}(s, x) .
$$

Usando o Lema 3.10, podemos escrever

$$
\tilde{u}_{x_{0}}(\tau, x)=\lim _{m \rightarrow+\infty} \Phi_{+} * g_{x_{0}, m, \gamma}(\tau, x) .
$$

Conforme a estratégia contida no dado físico do problema, isto é, de se tomar a temperatura no mesmo ponto onde foi aplicada a carga térmica, fazemos

$$
x_{0}=x,
$$

para obter

$$
\begin{aligned}
\Phi_{+} * g_{x, m, \gamma}(\tau, x)= & \int_{0}^{\tau} \frac{1}{4 \pi} t \int_{|\xi|=1} \frac{(\tau-t)(-\imath)}{2 \pi} \int_{\gamma-\imath m}^{\gamma+\imath m} \frac{-2 p^{2}}{\pi}[3 \rho(x-t \xi)+ \\
& \left.t \frac{\partial \rho}{\partial r}(x-t \xi)-2 t^{2} p \rho(x-t \xi)\right] \\
& \exp \left[-p t^{2}\right] \exp \left[(\tau-t)^{2} p\right] \mathrm{d} p \mathrm{~d} \sigma_{\xi} \mathrm{d} t
\end{aligned}
$$

Definimos agora uma integração na esfera unitária.

Definição 3.2. Integração de $\rho$ na esfera unitária

$$
f(x, r) \doteq \int_{|\xi|=1} \rho(x-\xi r) \mathrm{d} \sigma_{\xi} .
$$

Em coordenadas polares,

$$
\frac{\partial f}{\partial r}(x, r)=\int_{|\xi|=1} \frac{\partial \rho}{\partial r}(x-\xi r) \mathrm{d} \sigma_{\xi} .
$$


Então, retornando ao cálculo da convolução:

$$
\begin{aligned}
\Phi_{+} * g_{x, m, \gamma}(\tau, x)= & \int_{0}^{\tau} \frac{1}{4 \pi^{2}} t(\tau-t) \frac{(-\imath)}{2 \pi} \int_{\gamma-\imath m}^{\gamma+\imath m}-2 p^{2}(3 f(x, t)+ \\
& \left.t \frac{\partial f}{\partial r}(x, t)-2 t^{2} p f(x, t)\right) \exp \left[p\left(-t^{2}+(\tau-t)^{2}\right)\right] \mathrm{d} p \mathrm{~d} t .
\end{aligned}
$$

Fazemos agora a mudança de variáveis

$$
t=\frac{\tau^{2}-z}{2 \tau}
$$

para obter

$$
\begin{aligned}
\Phi_{+} * g_{x, m, \gamma}(\tau, x)= & \int_{-\tau^{2}}^{\tau^{2}} \frac{1}{2 \tau} \frac{1}{4 \pi^{2}}\left(\frac{\tau^{2}-z}{2 \tau}\right)\left(\tau-\frac{\tau^{2}-z}{2 \tau}\right) \times \\
& {\left[3 f\left(x, \frac{\tau^{2}-z}{2 \tau}\right)+\left(\frac{\tau^{2}-z}{2 \tau}\right) \frac{\partial f}{\partial r}\left(x, \frac{\tau^{2}-z}{2 \tau}\right)\right] \times } \\
& \frac{(-\imath)}{2 \pi} \int_{\gamma-\imath m}^{\gamma+\imath m}(-2) p^{2} \exp [p z] \mathrm{d} p \mathrm{~d} z- \\
& \int_{-\tau^{2}}^{\tau^{2}} \frac{1}{2 \tau} \frac{1}{4 \pi^{2}}\left(\frac{\tau^{2}-z}{2 \tau}\right)\left(\tau-\frac{\tau^{2}-z}{2 \tau}\right) \times \\
& \left(\frac{\tau^{2}-z}{2 \tau}\right)^{2} f\left(x, \frac{\tau^{2}-z}{2 \tau}\right) \frac{(-\imath)}{2 \pi} \int_{\gamma-\imath m}^{\gamma+\imath m}(-4) p^{3} \exp [p z] \mathrm{d} p \mathrm{~d} z .
\end{aligned}
$$

Dai, usando o Lema 3.21, temos para $\tau>0$ e $x \in \Omega^{*}$,

$$
\tilde{u}_{x}(\tau, x)=\frac{1}{256 \pi^{2} \tau^{2}}\left[16 \frac{\partial f}{\partial r}\left(x, \frac{\tau}{2}\right)-8 \tau \frac{\partial^{2} f}{\partial r^{2}}\left(x, \frac{\tau}{2}\right)-\tau^{2} \frac{\partial^{3} f}{\partial r^{3}}\left(x, \frac{\tau}{2}\right)\right] .
$$

Como no caso $n=1$, para cada $x \in \Omega^{*}$ fixo, $\tilde{u}_{x}(x, \tau)$ é uma distribuição compactamente suportada.

Agora tratamos do caso $n \geq 5, n$ ímpar.

Como nos casos $n=1$ e $n=3$, partindo de

$$
\tilde{u}_{x_{0}}(\tau, x)=\Phi_{+} * g_{x_{0}}(\tau, x)=\lim _{m \rightarrow+\infty} \Phi_{+} * g_{x_{0}, m, \gamma},
$$

com $g_{x_{0}, m, \gamma}$ dada por (3.24), obtemos a fórmula

$$
\begin{aligned}
\tilde{u}_{x_{0}}(\tau, x)= & \lim _{m \rightarrow+\infty} \frac{\imath}{\sqrt{\pi} \omega_{n}(1.3 \ldots(n-2))} \int_{0}^{\tau} t \int_{\gamma-\imath m}^{\gamma+\imath m} \frac{\exp \left[p t^{2}\right]}{\sqrt{p}} \times \\
& \frac{1}{\pi^{n / 2}} p^{1+n / 2}\left\{\left(\frac{1}{r} \frac{\partial}{\partial r}\right)^{\frac{n-3}{2}} E(r, x, p)\right\}_{r=\tau-t} \mathrm{~d} p \mathrm{~d} t
\end{aligned}
$$


em que

$$
E(r, x, p)=\frac{-\pi^{n / 2}}{2 p^{1+n / 2}} r^{n-2} \int_{\partial B_{1}(x)} \operatorname{div}\left(\rho \vec{\nabla} \dot{u}_{x_{0}}\right)\left(\frac{1}{4 p}, x-r \xi\right) \mathrm{d} S_{\xi},
$$

e $\omega_{n}$ é a medida da esfera unitária em $\mathbb{R}^{\mathrm{n}}$.

Na Fórmula (3.27), usamos $x_{0}=x$, isto é, o mesmo ponto onde $u$ é medido.

Após substituir (3.13) em (3.28), e usar (3.25) temos

$$
\begin{aligned}
E(r, x, p) & =\exp \left[-p r^{2}\right] \times \\
& \left(3 r^{n-2} f(x, r)-2 r^{n} p f(x, r)+r^{n-1} \frac{\partial f}{\partial r}(x, r)\right) .
\end{aligned}
$$

Usando a expansão binomial, vemos que o termo $\left(\frac{1}{r} \frac{\partial}{\partial r}\right)^{\frac{n-3}{2}} E(r, x, p)$ que aparece em (3.27) pode ser escrito como

$$
\begin{aligned}
\left(\frac{1}{r} \frac{\partial}{\partial r}\right)^{\frac{n-3}{2}} E(r, x, p)= & \exp \left[-p r^{2}\right] \times \\
\sum_{j=0}^{\frac{n-3}{2}}[ & P_{1}(p, r, j) \partial_{r}^{\frac{n-3}{2}-j} f(x, r)+ \\
& P_{2}(p, r, j+1) \partial_{r}^{\frac{n-3}{2}-j} f(x, r)+ \\
& \left.P_{3}(p, r, j) \partial_{r}^{\frac{n-3}{2}-j+1} f(x, r)\right],
\end{aligned}
$$

em que $P_{k}(p, r, j), k=1,2,3$ são polinômios em $p$ de ordem $j$, que não contém $f$ nem suas derivadas. Todos os coeficientes dependem somente de potências positivas de $r$. 
Substituindo (3.30) em (3.27), obtemos,

$$
\begin{aligned}
\tilde{u}_{x}(\tau, x)= & \lim _{m \rightarrow+\infty} \frac{\imath}{\sqrt{\pi} \omega_{n}(1.3 \ldots(n-2)) \pi^{n / 2}} \int_{0}^{\tau} t \times \\
& \int_{\gamma-\imath m}^{\gamma+\imath m} p^{(n+1) / 2}\left\{\exp \left[p\left(t^{2}-r^{2}\right)\right] \times\right. \\
& \left.\sum_{j=0}^{\frac{n-3}{2}} P_{1}(p, r, j) P_{2}\left(f, x, r, p, \frac{n-3}{2}-j+1\right)\right\} \quad-2 \\
= & \lim _{m \rightarrow+\infty} \frac{-2}{\omega_{n}(1.3 \ldots(n-2)) \pi^{(n-1) / 2}} \int_{0}^{\tau} t \times \\
& \frac{-\imath}{2 \pi} \int_{\gamma-\imath m}^{\gamma+\imath m} p^{(n+1) / 2} \exp \left[p\left(t^{2}-(\tau-t)^{2}\right)\right] \times \\
& \sum_{j=0}^{\frac{n-3}{2}}\left[P_{1}(p, r, j) \partial_{r}^{\frac{n-3}{2}-j} f(x, r)+P_{2}(p, r, j+1) \partial_{r}^{\frac{n-3}{2}-j} f(x, r)+\right. \\
& \left.P_{3}(p, r, j) \partial_{r}^{\frac{n-3}{2}-j+1} f(x, r)\right] \mathrm{d} p \mathrm{~d} t
\end{aligned}
$$

Fazendo a mudança de variáveis

$$
s=t^{2}-(\tau-t)^{2}
$$

para $\tau>0$ em (3.31), ela se transforma em

$$
\begin{aligned}
\tilde{u}_{x}(\tau, x)= & \lim _{m \rightarrow+\infty} \frac{-1}{\tau \omega_{n}(1.3 \ldots(n-2)) \pi^{(n-1) / 2}} \int_{-\tau^{2}}^{\tau^{2}}\left(\frac{s+\tau^{2}}{2 \tau}\right) \frac{-\imath}{2 \pi} \times \\
& \int_{\gamma-\imath m}^{\gamma+\imath m} p^{(n+1) / 2} \exp [p s] \times \\
& \sum_{j=0}^{\frac{n-3}{2}}\left[P_{1}(p, r, j) \partial_{r}^{\frac{n-3}{2}-j} f(x, r)+P_{2}(p, r, j+1) \partial_{r}^{\frac{n-3}{2}-j} f(x, r)+\right. \\
& \left.P_{3}(p, r, j) \partial_{r}^{\frac{n-3}{2}-j+1} f(x, r)\right] \mathrm{d} p \mathrm{~d} s
\end{aligned}
$$

em uma vizinhança de $\tau=0$, temos simplesmente que $\tilde{u}=0$, quando $x \in \Omega^{*}$.

Aplicando o Lema 3.21 e lembrando a natureza de $P_{k}, k=1,2,3$, vemos que para $x \in \mathbb{R}^{\mathrm{n}}$ fixo, (3.32) representa uma equação para a função $r \mapsto f(x, r)$. A ordem dessa equação é dada pela ordem da derivação em $r$ mais alta atingida. Não se esquecendo das derivações obtidas pela transformada inversa de Laplace, temos que a ordem é $n$.

Para $\tau$ suficientemente grande, ou mais precisamente, para $\tau>\operatorname{diam}\left(\Omega \bigcup \Omega^{*}\right), f(x, \tau)=$ $0, \forall x \in \Omega^{*}$. Isso significa que se $u(\tau, x)=0$ para $\tau \in[0, T], T>2 \operatorname{diam}\left(\Omega \bigcup \Omega^{*}\right), \forall x \in \Omega^{*}$, 
então a equa(3.32) com condições iniciais nulas em $T$ implica que $f(x, r)=0, \forall r>0$, $\forall x \in \Omega^{*}$.

Nesse trabalho, especializamos os resultados para $n=1$ e $n=3$, casos importantes em aplicações industriais. As conclusões a respeito da unicidade para a recuperação de $\rho$ para $n \geq 5$ ímpar são as mesmas, se levarmos em conta (3.32) e as observações feitas no último parágrafo.

Para concluir essa seção, reapresentamos as equações resultantes para os casos $n=1 \mathrm{e}$ $n=3$.

Para $n=1$ temos $\forall x \in \Omega^{*}$ :

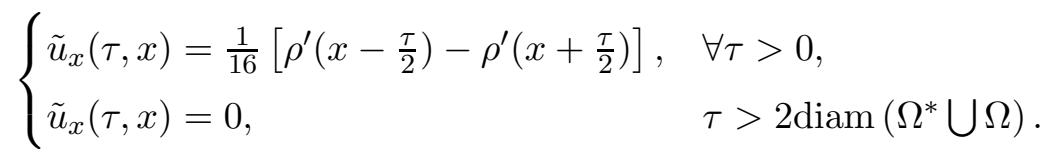

Para $n=3$, temos $\forall x \in \Omega^{*}$ :

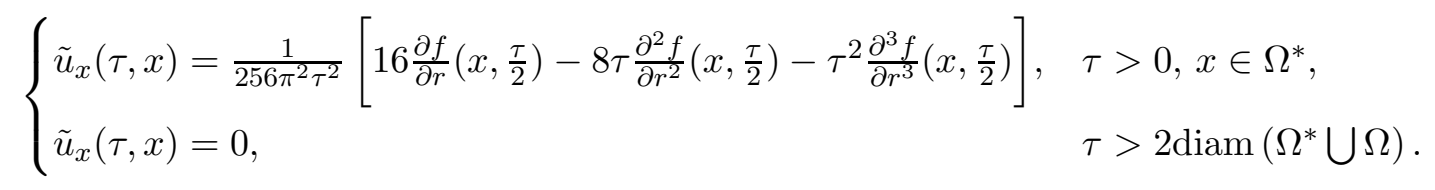

Note que as expressões acima mostram que para cada $x \in \Omega^{*}$, a função $\tau \mapsto \tilde{u}_{x}(\tau, x)$ é compactamente suportada. Além disso, pelas discussões anteriores, $\forall x \in \Omega^{*}, \tilde{u}_{x}(\tau, x)$ se anula para $\tau$ em uma vizinhança de $\{0\}$ em $[0,+\infty)$.

Para relembrar, nosso problema é a determinação unívoca da função $\rho \in \mathcal{C}^{n}\left(\mathbb{R}^{\mathrm{n}}\right)$ que tem suporte em $\Omega \operatorname{com} \bar{\Omega} \cap \overline{\Omega^{*}}=\emptyset$.

\subsection{Transformação do dado sintetizado em $\Gamma_{\Omega^{*}}$}

O problema linearizado (3.2) foi transformado na equação hiperbólica (3.4). É importante saber o que ocorre com o dado do problema inverso

$$
\Gamma_{\Omega^{*}}=\left\{u_{x}(t, x) \mid x \in \Omega^{*}, a<t<b\right\},
$$

em que $0<a<b$.

Lema 3.22. Se $\Gamma_{\Omega^{*}}=\{0\}$, então $\tilde{u}_{x}(\tau, x)=0$ como um elemento de $\mathcal{D}^{\prime}\left([0,+\infty) \times \Omega^{*}\right)$.

Demonstração. Como $u_{x}(t, x)={ }^{t} \mathcal{H}\left(\tilde{u}_{x}\right)(t, x)$, temos para qualquer $\psi \in \mathcal{C}_{c}^{\infty}\left(\Omega^{*}\right)$ e $a<t<$ $b$ :

$$
0=\left\langle\tilde{u}_{x}(\tau, x), \exp \left[\frac{-\tau^{2}}{4 t}\right] \psi(x)\right\rangle_{t, x} .
$$


Agora usamos o difeomorfismo $b:(s, x) \mapsto\left(2 \sqrt{T_{0}} s, x\right)$, em que $\left.T_{0} \in\right] a, b[$, para obter

$$
0=\left\langle b^{*} \tilde{u}_{x}(s, x), \exp \left[\frac{-s^{2} T_{0}}{t}\right] \psi(x)\right\rangle_{s, x} .
$$

$\operatorname{Em} t=T_{0}$, (3.33) fornece $\left\langle b^{*} \tilde{u}_{x}(s, x), \exp \left[-s^{2}\right] \psi(x)\right\rangle_{s, x}=0$.

Derivando (3.33) com respeito a $t$, e calculando o resultado em $t=T_{0}$, obtemos

$$
\left\langle b^{*} \tilde{u}_{x}(s, x), s^{2} \exp \left[-s^{2}\right] \psi(x)\right\rangle_{s, x}=0 .
$$

Iterando o argumento, temos

$$
\left\langle b^{*} \tilde{u}_{x}(s, x), s^{2 k} \exp \left[-s^{2}\right] \psi(x)\right\rangle_{s, x}=0, \forall k \in \mathbb{Z}_{+} .
$$

Por outro lado, como $\rho$ tem suporte compacto, temos pelas fórmulas explícitas de $\tilde{u}_{x}$ que existe $\tau^{*}>0$ tal que se $\phi$ é qualquer função $\left.\left.\operatorname{com} \operatorname{supp}(\phi) \subset\right] \tau^{*},+\infty\right)$, então

$$
\left\langle b^{*} \tilde{u}_{x}(s, x), \phi(s) \psi(x)\right\rangle_{s, x}=0 .
$$

Pelo Teorema de Stone-Weierstras,

$$
\left\{P\left(s^{2}\right) \exp \left[-s^{2}\right] \mid P \text { é uma função polinomial }\right\}
$$

é denso no conjunto das funções teste compactamente suportadas em $\left[0, \tau^{*}\right]$. Isso termina a prova.

\subsection{Prova da unicidade para a recuperação de $\rho$}

Devemos considerar dois casos. O primeiro é para $n=1$.

Proposição 3.23. Seja $T=3 \operatorname{diam}\left(\Omega \bigcup \Omega^{*}\right)$. Se $\tilde{u}_{x}(\tau, x)=0$ em $\left.] 0, T\right] \times \Omega^{*}$ então $\rho \equiv 0$.

Demonstração. Como $\rho \in \mathcal{C}^{1}$ tem suporte compacto, basta provar que $\rho^{\prime} \equiv 0$. Iremos provar que para qualquer $\delta>0$ arbitrariamente pequeno, não existe nenhum intervalo de comprimento maior que $\delta$ em que $\rho^{\prime}$ nunca se anula.

Podemos supor que $\delta$ é suficientemente pequeno tal que exista $x \in \Omega^{*}$ de modo que $\{x-\delta / 2, x+\delta / 2\} \subset \Omega^{*}$.

Seja $\tau_{1}=T$ e defina $m=\max \left\{i \in \mathbb{N} \mid \frac{\tau_{1}}{\delta}>2 i+1\right\}$.

Note que $m \geq 1$, pois $\tau_{1}=3 \operatorname{diam}\left(\Omega \bigcup \Omega^{*}\right)$ e $0<\delta<\operatorname{diam}\left(\Omega^{*}\right)$. 
Temos

$$
\begin{aligned}
& 0=\tilde{u}_{x-\frac{\delta}{2}}\left(\tau_{1}-\delta, x-\frac{\delta}{2}\right)=\frac{1}{16}\left[\rho^{\prime}\left(x-\frac{\tau_{1}}{2}\right)-\rho^{\prime}\left(x+\frac{\tau_{1}}{2}-\delta\right)\right], \\
& 0=\tilde{u}_{x+\frac{\delta}{2}}\left(\tau_{1}-\delta, x+\frac{\delta}{2}\right)=\frac{1}{16}\left[\rho^{\prime}\left(x-\frac{\tau_{1}}{2}+\delta\right)-\rho^{\prime}\left(x+\frac{\tau_{1}}{2}\right)\right], \\
& 0=\tilde{u}_{x}\left(\tau_{1}, x\right)=\frac{1}{16}\left[\rho^{\prime}\left(x-\frac{\tau_{1}}{2}\right)-\rho^{\prime}\left(x+\frac{\tau_{1}}{2}\right)\right] .
\end{aligned}
$$

De (3.34) e (3.36) obtemos

$$
\rho^{\prime}\left(x+\frac{\tau_{1}}{2}-\delta\right)=\rho^{\prime}\left(x+\frac{\tau_{1}}{2}\right)=0 .
$$

De (3.35) e (3.36) obtemos

$$
\rho^{\prime}\left(x-\frac{\tau_{1}}{2}+\delta\right)=\rho^{\prime}\left(x-\frac{\tau_{1}}{2}\right)=0 .
$$

É claro que os pontos de $\Omega$ podem ser divididos em subconjuntos à direita e à esquerda de $x$. Provamos a proposição para o subconjunto à direita. $\mathrm{O}$ argumento para o outro caso é simétrico.

O ponto $x+\frac{\tau_{1}}{2}$ está certamente fora do suporte de $\rho$, e a distância entre $x+\frac{\tau_{1}}{2} \mathrm{e} x+\frac{\tau_{1}}{2}-\delta$ é $\delta$. Mostraremos agora que a distância entre $x+\frac{\tau_{1}}{2}-\delta$ e o conjunto $\{x-\delta / 2, x+\delta / 2\}$ é no máximo $\delta$.

Se $m=1$, temos $\tau_{1}>3 \delta$ e $\tau_{1} \leq 5 \delta$. Isso significa que $\left.\left.\frac{\tau_{1}}{2} \in\right] \frac{3 \delta}{2}, \frac{5 \delta}{2}\right]$, e então a distância entre $x+\frac{\tau_{1}}{2}-\delta$ e o ponto $x+\delta / 2$ é no máximo $\delta$.

Procedemos por indução. Chame $\tau_{j}=\tau_{j-1}-2 \delta=\tau_{1}-2(j-1) \delta$, e assuma que, para $j \leq m+1$, temos

$$
\rho^{\prime}(x+\underbrace{\frac{\tau_{1}}{2}-(j-1) \delta}_{\frac{\tau_{j}}{2}})=\rho^{\prime}\left(x+\frac{\tau_{1}}{2}\right),
$$

o que certamente é verdade para $j-1=1$, como vimos anteriormente.

Como $j \leq m+1$, temos $\tau_{j}-\delta>0$ e dai

$$
\begin{aligned}
& 0=\tilde{u}_{x-\frac{\delta}{2}}\left(\tau_{j}-\delta, x-\frac{\delta}{2}\right)=\frac{1}{16}\left[\rho^{\prime}\left(x-\frac{\tau_{j}}{2}\right)-\rho^{\prime}\left(x+\frac{\tau_{j}}{2}-\delta\right),\right] \\
& 0=\tilde{u}_{x}\left(\tau_{j}, x\right)=\frac{1}{16}\left[\rho^{\prime}\left(x-\frac{\tau_{j}}{2}\right)-\rho^{\prime}\left(x+\frac{\tau_{j}}{2}\right)\right],
\end{aligned}
$$

donde concluímos que

$$
\rho^{\prime}\left(x+\frac{\tau_{j}}{2}-\delta\right)=\rho^{\prime}\left(x+\frac{\tau_{j}}{2}\right) .
$$

Agora, usando a hipótese de indução, obtemos

$$
\rho^{\prime}\left(x+\frac{\tau_{1}}{2}-j \delta\right)=\rho^{\prime}(x+\underbrace{\frac{\tau_{j}}{2}-\delta}_{\frac{\tau_{j+1}}{2}})=\rho^{\prime}\left(x+\frac{\tau_{1}}{2}\right) .
$$




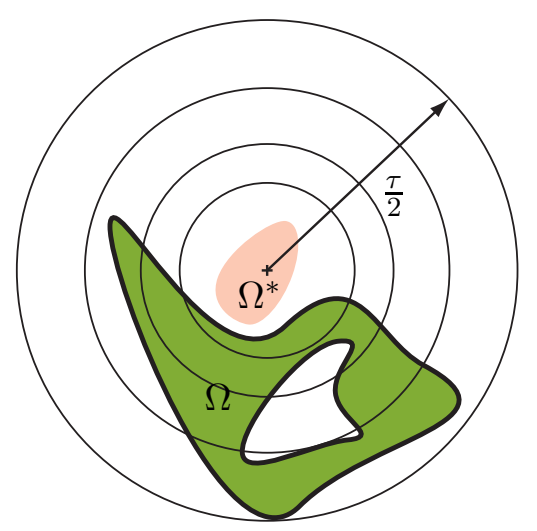

Figura 3.3: Aplicação de médias esféricas.

Finalmente, resta provar que a distância entre o ponto $x+\frac{\tau_{m+1}}{2}-\delta$ gerado na última iteração e o conjunto $\{x-\delta / 2, x+\delta / 2\}$ é no máximo $\delta$.

Pela definição de $m$, temos $\tau_{m+1}>\delta$ e $\tau_{m+1} \leq 3 \delta$, donde concluímos que $\frac{\tau_{m+1}}{2} \in$ ]$\delta / 2,3 \delta / 2]$. Portanto a distância entre $x+\frac{\tau_{m+1}}{2}-\delta$ e o ponto $x+\delta / 2$ é no máximo $\delta$.

O outro caso, $n>1$, requer uma análise de integrais em esferas.

\subsubsection{O uso de médias esféricas}

Nesta seção, provamos a unicidade no caso $n=3$ empregando médias esféricas e um teorema devido a Zalcman [14].

Primeiramente, é importante reinterpretarmos a Equação (3.26). Para cada $\tau>0$, o lado direito de (3.26) fornece uma extensão natural de $x \mapsto \tilde{u}_{x}(\tau, x)$ para todo o $\mathbb{R}^{3}$. Chame esta extensão de $\tilde{U}_{x}(\tau, x)$. Então

$$
\tilde{U}_{x}(\tau, x)=\frac{1}{256 \pi^{2} \tau^{2}}\left[16 \frac{\partial f}{\partial r}\left(x, \frac{\tau}{2}\right)-8 \tau \frac{\partial^{2} f}{\partial r^{2}}\left(x, \frac{\tau}{2}\right)-\tau^{2} \frac{\partial^{3} f}{\partial r^{3}}\left(x, \frac{\tau}{2}\right)\right], \forall \tau>0, \forall x \in \mathbb{R}^{\mathrm{n}} .
$$

Note que, embora a função original $\tilde{u}_{x}(\tau, x)$ seja definida somente para $\Omega^{*}$, nós devemos integrar $\rho$ em esferas que varrem $\Omega$. Esta situação está ilustrada esquematicamente na Figura 3.3.

Para concluirmos que $\rho \equiv 0$, devemos analisar suas médias esféricas. 


\section{Médias esféricas}

Definimos a média esférica de $\tilde{U}(\tau, \xi) \doteq \tilde{U}_{\xi}(\tau, \xi)$ tomada sobre a esfera de centro $x \in \mathbb{R}^{\mathrm{n}} \mathrm{e}$ raio $R$ por

$$
J(x, R, \tau)=\frac{1}{\omega_{n}} \int_{\partial B_{1}(0)} \tilde{U}(\tau, x+R \xi) \mathrm{d} S_{\xi},
$$

em que $\omega_{n}$ é a medida da média esférica em $\mathbb{R}^{\mathrm{n}}$, e denotamos por $B_{r}(y) \subset \mathbb{R}^{\mathrm{n}}$ a bola de raio $r$ centrada em $y$.

Definimos a média esférica iterada de $\rho$ em torno de um ponto $x$ por

$$
\begin{aligned}
& M\left(x, R_{1}, R_{2}\right) \quad \doteq \frac{1}{\omega_{n}} \int_{\partial B_{1}(0)} f\left(x+R_{2} \zeta, R_{1}\right) \mathrm{d} S_{\zeta}= \\
& =\frac{1}{\omega_{n}} \int_{\partial B_{1}(0)} \int_{\partial B_{1}(0)} \rho\left(x-R_{1} \xi+R_{2} \zeta\right) \mathrm{d} S_{\xi} \mathrm{d} S_{\zeta} .
\end{aligned}
$$

No caso do $\mathbb{R}^{3}$, dividimos (3.37) por $\omega_{3}=4 \pi$ e a integramos sobre a esfera de raio $R \geq 0$, para obter

$$
J(x, R, \tau)=\left\{\begin{array}{cc}
\frac{1}{4 \pi} \int_{\partial B_{1}(0)} \tilde{U}(\tau, x+R \xi) \mathrm{d} S_{\xi}= \\
\frac{1}{256 \pi^{2} \tau^{2}}\left[16 \frac{\partial M}{\partial R_{1}}\left(x, \frac{\tau}{2}, R\right)-8 \tau \frac{\partial^{2} M}{\partial R_{1}^{2}}\left(x, \frac{\tau}{2}, R\right)-\tau^{2} \frac{\partial^{3} M}{\partial R_{1}^{3}}\left(x, \frac{\tau}{2}, R\right)\right] \\
\tau>0, R>0, x \in \mathbb{R}^{3} \\
\tilde{U}_{x}(\tau, x), & \tau>0, R=0, x \in \mathbb{R}^{3} .
\end{array}\right.
$$

Para o próximo lema, precisaremos do Teorema de Green. É fato bem conhecido que para uma função $h: \mathbb{R}^{\mathrm{n}} \rightarrow \mathbb{R}$ de Classe $\mathcal{C}^{1}$ vale:

$$
\frac{\partial}{\partial \dot{x}_{i}} \int_{B_{R}(0)} h(\dot{x}+x) \mathrm{d} x=\int_{B_{R}(0)} \frac{\partial}{\partial x_{i}} h(\dot{x}+x) \mathrm{d} x=\int_{\partial B_{R}(0)} h(\dot{x}+x) \nu_{i} \mathrm{~d} S_{x},
$$

em que $\nu_{i}$ é a i-ésima componente do vetor normal exterior à $\partial B_{R}(0)$.

Mas a fórmula obtida com os dois extremos da igualdade acima,

$$
\frac{\partial}{\partial \dot{x}_{i}} \int_{B_{R}(0)} h(\dot{x}+x) \mathrm{d} x=\int_{\partial B_{R}(0)} h(\dot{x}+x) \nu_{i} \mathrm{~d} S_{x},
$$

vale também para o caso de $h$ ser apenas contínua.

Isso pode ser visto pelo argumento exposto a seguir. Tome $U \subset \mathbb{R}^{\mathrm{n}}$ um aberto limitado arbitrário tal que $\dot{x} \in U$. Então existe um compacto $K$ tal que se $\dot{x} \in U$ e $x \in B_{R}(\dot{x})$, então $x+\dot{x} \in K$.

Pelo Teorema de Stone-Weierstrass, existe uma seqüência de polinômios $\left\{h_{j}\right\}_{j}$ tal que

$$
h_{j} \underset{j \rightarrow+\infty}{\stackrel{\text { unif. }}{\longrightarrow}} h \quad \text { em } K .
$$


Então de (3.40), com $h_{j}$ de classe $\mathcal{C}^{\infty}$, temos

$$
\frac{\partial}{\partial \dot{x}_{i}} \int_{B_{R}(0)} h_{j}(\dot{x}+x) \mathrm{d} x=\int_{\partial B_{R}(0)} h_{j}(\dot{x}+x) \nu_{i} \mathrm{~d} S_{x} .
$$

Mas

$$
\int_{\partial B_{R}(0)} h_{j}(\dot{x}+x) \nu_{i} \mathrm{~d} S_{x} \underset{j \rightarrow+\infty}{\stackrel{\text { unif. }}{\longrightarrow}} \int_{\partial B_{R}(0)} h(\dot{x}+x) \nu_{i} \mathrm{~d} S_{x},
$$

para $\dot{x} \in U$.

Concluimos então que a seqüência

$$
\left\{\frac{\partial}{\partial \dot{x}_{i}} \int_{B_{R}(0)} h_{j}(\dot{x}+x) \mathrm{d} x\right\}_{j}
$$

converge uniformemente para $\dot{x} \in U$, e portanto da teoria clássica temos que

$$
\begin{aligned}
\frac{\partial}{\partial \dot{x}_{i}} \int_{B_{R}(0)} h(\dot{x}+x) \mathrm{d} x & =\frac{\partial}{\partial \dot{x}_{i}} \lim _{j \rightarrow+\infty} \int_{B_{R}(0)} h_{j}(\dot{x}+x) \mathrm{d} x= \\
& =\lim _{j \rightarrow+\infty} \frac{\partial}{\partial \dot{x}_{i}} \int_{B_{R}(0)} h_{j}(\dot{x}+x) \mathrm{d} x= \\
& =\int_{\partial B_{R}(0)} h(\dot{x}+x) \nu_{i} \mathrm{~d} S_{x} .
\end{aligned}
$$

Vemos então que, de fato, (3.40) é verdadeira para $h$ apenas contínua.

Lema 3.24. Seja $D \subset \mathbb{R}^{\mathrm{n}}$ aberto e conexo. Seja também $\left.\left.h: D \times\right] 0,+\infty\right) \rightarrow \mathbb{R}$ tal que, $\forall p>0, x \mapsto h(x, p)$ é contínua em $D$.

Seja $\dot{x}=\left(\dot{x}_{1}, \dot{x}_{2}, \ldots, \dot{x}_{n}\right) \in D$, e tome $R>0$ tal que $|x-\dot{x}| \leq R$ implica $x \in D$.

Então

$$
\begin{aligned}
\int_{\partial B_{1}(0)} h(\dot{x}+\xi R, p)\left(\dot{x}_{i}+\xi_{i} R\right) \mathrm{d} S_{\xi}=\dot{x}_{i} \int_{\partial B_{1}(0)} h(\dot{x}+\xi R, p) \mathrm{d} S_{\xi}+ \\
\\
\quad \frac{1}{R^{n-2}} \frac{\partial}{\partial \dot{x}_{i}} \int_{0}^{R} \int_{\partial B_{1}(0)} h(\dot{x}+\xi r, p) \mathrm{d} S_{\xi} r^{n-1} \mathrm{~d} r, \forall p>0, \forall i=1, \ldots, n .
\end{aligned}
$$

Demonstração. Pelo Teorema de Green, que acabamos de discutir, temos $\forall p>0$ :

$$
\frac{\partial}{\partial \dot{x}_{i}} \int_{B_{R}(0)} h(\dot{x}+x, p) \mathrm{d} x=\int_{\partial B_{R}(0)} h(\dot{x}+x, p) \nu_{i} \mathrm{~d} S,
$$

em que $\nu_{i}$ é a i-ésima componente do vetor normal exterior à $\partial B_{R}(0)$.

Escrevendo a equação acima em coordenadas polares e dividindo o resultado por $R^{n-2}$, obtemos

$$
\frac{1}{R^{n-2}} \frac{\partial}{\partial \dot{x}_{i}} \int_{0}^{R} \int_{\partial B_{1}(0)} h(\dot{x}+\xi r, p) \mathrm{d} S_{\xi} r^{n-1} \mathrm{~d} r=\int_{\partial B_{1}(0)} h(\dot{x}+\xi R, p) R \xi_{i} \mathrm{~d} S_{\xi}, \forall p>0 .
$$

Somando a ambos os lados $\dot{x}_{i} \int_{\partial B_{1}(0)} h(\dot{x}+\xi R, p) \mathrm{d} S_{\xi}$, obtemos o resultado desejado. 
O próximo lema, que é fundamental para o trabalho, é uma versão com parâmetros de um resultado devido a Zalcman [14].

Lema 3.25. Seja $D$ como no lema anterior, e $h \in \mathcal{C}(D \times] 0,+\infty))$. Suponha que $\forall x \in D e$ $\forall R>0$ tal que $x+\xi R \in D, \forall \xi \in \partial B_{1}(0)$ seja verdade que

$$
h(x, p)=0, \forall p>0 \Rightarrow \int_{\partial B_{1}(0)} h(x+\xi R, p) \mathrm{d} S_{\xi}=0, \forall p>0 .
$$

Então a existência de um aberto $U \neq \emptyset$ tal que $h(x, p)=0, \forall x \in U, \forall p>0$, implica em $h(x, p)=0, \forall p>0, \forall x \in D$.

Demonstração. Seja o conjunto

$$
\mathcal{U}=\left\{x \in D \mid \exists V \subset D \text { aberto tal que } x \in V \text { e }\left.h\right|_{V \times] 0,+\infty)}=0 .\right\}
$$

Note que $\mathcal{U}$ é aberto por definição e $\mathcal{U} \neq \emptyset$ por hipótese, pois $U \subset \mathcal{U}$.

A hipótese também implica que para $x_{0} \in \mathcal{U}$ e $\forall R>0$ tal que $B_{R}\left(x_{0}\right) \subset D$, temos

$$
\int_{\partial B_{1}(0)} h\left(x_{0}+\xi R, p\right) \mathrm{d} S_{\xi}=0, \forall p>0, \forall i=1, \ldots n .
$$

O Lema 3.24 implica que a função $\left.\left.g_{i}: D \times\right] 0,+\infty\right) \rightarrow \mathbb{R}$ definida por $g_{i}(x, p)=$ $h(x, p) x_{i}, i=1, \ldots, n$, satisfaz

$$
\int_{\partial B_{1}(0)} g_{i}\left(x_{0}+\xi R, p\right) \mathrm{d} S_{\xi}=0, \forall x_{0} \in \mathcal{U}, \forall R>0, B_{R}\left(x_{0}\right) \subset D, \forall p>0,
$$

e conseqüentemente

$$
\int_{\partial B_{1}(0)} h\left(x_{0}+\xi R, p\right) \mathbb{P}\left(x_{0}+\xi R\right) \mathrm{d} S_{\xi}=0, \forall x_{0} \in \mathcal{U}, \forall R>0, B_{R}\left(x_{0}\right) \subset D, \forall p>0,
$$

para toda função polinomial $\mathbb{P}(x)$.

Pelo Teorema de Stone-Weierstrass, podemos aproximar uniformemente $h(\cdot, p)$, para cada $p>0$ fixado, por uma seqüência de polinômios em $\partial B_{R}\left(x_{0}\right)$. Portanto

$$
\int_{\partial B_{1}(0)} h^{2}\left(x_{0}+\xi R, p\right) \mathrm{d} S_{\xi}=0, \forall p>0 .
$$

Desse fato segue que $\mathcal{U}$ é fechado em $D$.

No teorema final deste trabalho, onde provamos a unicidade na recuperação de $\rho$, provaremos que para todo $x_{0} \in \mathbb{R}^{3}, \tau>0, \tilde{U}_{x_{0}}\left(\tau, x_{0}\right)=0$ implica que a média esférica de $\tilde{U}\left(\tau, x_{0}\right) \doteq \tilde{U}_{x_{0}}\left(\tau, x_{0}\right)$, dada por (3.38) é zero, isto é, $J\left(x_{0}, R, \tau\right)=0, \forall R>0, \forall \tau>0$. Dai a unicidade da recuperação de $\rho$ será uma conseqüência do Lema 3.25, pois de (3.3) e do Lema 3.22 sabemos que $\tilde{U}_{x}(\tau, x)=0, \forall \tau>0, \forall x \in \Omega^{*}$. 
Teorema 3.26 (Unicidade na recuperação de $\rho$ ). Se $\tilde{U}_{x}(\tau, x)=0, \forall x \in \Omega^{*}, \forall \tau>0$ então $\rho \in \mathcal{C}^{3}\left(\mathbb{R}^{3}\right)$ com $\operatorname{supp}(\rho) \subset \Omega$ é nula.

Demonstração. Aplicaremos o Lema 3.25. Para isso, fixamos $x_{0} \in \mathbb{R}^{3}$, e supomos que $\tilde{U}_{x_{0}}\left(\tau, x_{0}\right)=0, \forall \tau>0$. De (3.37), após fazermos a substituição $\frac{\tau}{2}=r$, obtemos

$$
4 \frac{\partial f}{\partial r}\left(x_{0}, r\right)-4 r \frac{\partial^{2} f}{\partial r^{2}}\left(x_{0}, r\right)-r^{2} \frac{\partial^{3} f}{\partial r^{3}}\left(x_{0}, r\right)=0, \forall r>0
$$

Como $\operatorname{supp}(\rho)$ é limitado, existe $r>0$ tal que $0=f\left(x_{0}, r\right)=\frac{\partial f}{\partial r}\left(x_{0}, r\right)=\frac{\partial^{2} f}{\partial r^{2}}\left(x_{0}, r\right)$. Portanto, pela unicidade das soluções de uma EDO, temos que $f\left(x_{0}, r\right)=0, \forall r>0$.

Agora aplicamos uma fórmula de [7] que relaciona médias esféricas e médias esféricas iteradas:

$$
\begin{aligned}
M\left(x_{0}, R_{1}, R_{2}\right)= & \frac{2 \omega_{n-1}}{\omega_{n}\left(2 R_{1} R_{2}\right)^{n-2}} \int_{R_{1}-R_{2}}^{R_{1}+R_{2}}\left[\left(r+R_{1}-R_{2}\right)\left(r+R_{1}+R_{2}\right) \times\right. \\
& \left.\left(R_{2}+r-R_{1}\right)\left(R_{2}-r+R_{1}\right)\right]^{(n-3) / 2} f\left(x_{0}, r\right) r \mathrm{~d} r,
\end{aligned}
$$

que para $n=3$ se torna

$$
M\left(x_{0}, R_{1}, R_{2}\right)=\frac{1}{2 \pi R_{1} R_{2}} \int_{R_{1}-R_{2}}^{R_{1}+R_{2}} f\left(x_{0}, r\right) r \mathrm{~d} r
$$

Lembrando que $f\left(x_{0}, r\right)=0, \forall r>0$, temos $M\left(x_{0}, R_{1}, R_{2}\right)=0, \forall R_{1}, R_{2}>0$. Portanto, de (3.39) vemos que $J\left(x_{0}, R, \tau\right)=0, \forall \tau>0, \forall R>0$.

Aplicando o Lema 3.25 , como $\left.\left.\tilde{U}_{x}(\tau, x)=0, \forall(\tau, x) \in\right] 0,+\infty\right) \times \Omega^{*}$, concluímos que $\tilde{U}_{x}(\tau, x)=0, \forall x \in \mathbb{R}^{3}, \tau>0$.

Conseqüentemente, de (3.37), obtemos $f(x, r)=0, \forall x \in \mathbb{R}^{3}, \forall r>0$, e então

$$
\int_{\partial B_{1}(0)} \rho(x+r \xi) \mathrm{d} S_{\xi}=0, \forall x \in \mathbb{R}^{3}, \forall r>0
$$

o que conclui a prova, já que $\rho$ é contínua. 


\section{Apêndice A}

\section{Enunciados de alguns teoremas}

\section{A.1 Resultados da teoria dos espaços de Sobolev}

Os resultados dessa seção são retirados de [4].

Proposição A.1. Seja $U$ um aberto limitado com fronteira de classe $\mathcal{C}^{1}$ e $m \in \mathbb{Z}_{+}$. Se

$$
\begin{aligned}
u & \in \mathrm{L}^{2}\left(0, T ; \mathrm{H}^{m+2}(U)\right), \\
u^{\prime} & \in \mathrm{L}^{2}\left(0, T ; \mathrm{H}^{m}(U)\right),
\end{aligned}
$$

então

$$
u \in \mathcal{C}^{0}\left([0, T], \mathrm{H}^{m+1}(U)\right)
$$

Proposição A.2. Seja $U \subset \mathbb{R}^{\mathrm{n}}$ um aberto limitado com fronteira de classe $\mathcal{C}^{1}$. Seja $u \in$ $\mathrm{H}^{k}(U), k \in \mathbb{Z}_{+}$. Se $2 k>n$, então

$$
u \in \mathcal{C}^{k-[n / 2]-1, \gamma}(\bar{U})
$$

com

$$
\gamma= \begin{cases}{[n / 2]+1-n / 2,} & \text { se } n / 2 \notin \mathbb{Z}_{+} \\ \text {Qualquer } \alpha \in] 0,1[, & \text { se } n / 2 \in \mathbb{Z}_{+}\end{cases}
$$

\section{A.2 Alguns resultados para a Equação do Calor}

\section{A.2.1 O conceito de solução fraca}

Os resultados dessa seção são retirados de [4].

Seja o operador

$$
\mathcal{L}(u)=\frac{\partial u}{\partial t}+\mathcal{M} u
$$


em que

$$
\mathcal{M} u=\sum_{i=1}^{n}\left[\sum_{j=1}^{n}\left(-a_{i j}(t, x) u_{x_{j}}\right)_{x_{i}}+b_{i}(t, x) u_{x_{i}}\right]+c(t, x) u,
$$

cujos os coeficientes pertencem aos seguintes espaços

$$
\begin{aligned}
a_{i j} & \in \mathrm{L}^{\infty}\left([0, T] \times \mathbb{R}^{\mathrm{n}}\right) \\
b_{i} & \in \mathrm{L}^{\infty}\left([0, T] \times \mathbb{R}^{\mathrm{n}}\right) \\
c & \in \mathrm{L}^{\infty}\left([0, T] \times \mathbb{R}^{\mathrm{n}}\right) .
\end{aligned}
$$

Ao operador $\mathcal{M}$, associamos a forma bilinear $B$ definida por

$$
\begin{aligned}
B[u, v ; t]= & \sum_{i=1}^{n}\left[\sum_{j=1}^{n} \int_{\mathbb{R}^{\mathrm{n}}} a_{i j}(t, x) u_{x_{j}}(t, x) v_{x_{i}}(t, x) \mathrm{d} x+\right. \\
& \left.\int_{\mathbb{R}^{\mathrm{n}}} b_{i}(t, x) u_{x_{i}}(t, x) v(t, x) \mathrm{d} x\right]+ \\
& \int_{\mathbb{R}^{\mathrm{n}}} c(t, x) u(t, x) v(t, x) \mathrm{d} x
\end{aligned}
$$

para todo $u, v$ em $\mathrm{H}^{1}\left(\mathbb{R}^{\mathrm{n}}\right)$ e quase todo $t \in[0, T]$.

Para $f \in \mathrm{L}^{2}\left(0, T ; \mathrm{L}^{2}\left(\mathbb{R}^{\mathrm{n}}\right)\right)$ e $g \in \mathrm{L}^{2}\left(\mathbb{R}^{\mathrm{n}}\right)$, a equação

$$
\begin{aligned}
\mathcal{L}(u) & =f, & & \text { em }] 0, T] \times \mathbb{R}^{\mathrm{n}} \\
u & =g, & & \text { em }\{0\} \times \mathbb{R}^{\mathrm{n}}
\end{aligned}
$$

tem solução fraca $u \in \mathrm{L}^{2}\left(0, T ; \mathrm{H}^{1}\left(\mathbb{R}^{\mathrm{n}}\right)\right), u^{\prime} \in \mathrm{L}^{2}\left(0, T ; \mathrm{H}^{-1}\left(\mathbb{R}^{\mathrm{n}}\right)\right)$ se $u$ satisfizer

i) Para quase todo $t \in[0, T]$,

$$
\left\langle u^{\prime}, v\right\rangle+B[u, v ; t]=(f, v), \forall v \in \mathrm{H}^{1}\left(\mathbb{R}^{\mathrm{n}}\right) ;
$$

ii) $u(0)=g$.

Observação A.1. Como $u \in \mathrm{L}^{2}\left(0, T ; \mathrm{H}^{1}\left(\mathbb{R}^{\mathrm{n}}\right)\right), u^{\prime} \in \mathrm{L}^{2}\left(0, T ; \mathrm{H}^{-1}\left(\mathbb{R}^{\mathrm{n}}\right)\right)$, sabemos que $u \in$ $\mathcal{C}^{0}\left([0, T], \mathrm{L}^{2}\left(\mathbb{R}^{\mathrm{n}}\right)\right)$, e assim, a condição (ii) faz sentido.

\section{A.2.2 Espaços de solução}

Os resultados dessa seção são retirados de [4]. 
Proposição A.3. Seja $U \subset \mathbb{R}^{\mathrm{n}}$ um aberto limitado com fronteira de classe $\mathcal{C}^{1}$. Considerando o mesmo operador da seção A.2.1, seja o problema

$$
\begin{cases}\mathcal{L}(u)=f, & \text { em }] 0, T] \times U, \\ u=0, & \text { em }[0, T] \times \partial U, \\ u=g, & \text { em }\{0\} \times U,\end{cases}
$$

com

$$
\begin{aligned}
g & \in \mathrm{H}^{2 m+1}(U), \\
\frac{\mathrm{d}^{k} f}{\mathrm{~d} t^{k}} & \in \mathrm{L}^{2}\left(0, T ; \mathrm{H}^{2 m-2 k}(U)\right), k=0, \ldots, m,
\end{aligned}
$$

e as condições de compatibilidade

$$
\begin{aligned}
g_{0} \doteq g \in \mathrm{H}_{0}^{1}(U) \\
g_{1} \doteq f(0)-\mathcal{M} g_{0} \in \mathrm{H}_{0}^{1}(U) \\
\vdots \\
g_{m} \doteq \\
\quad \frac{\mathrm{d}^{m-1}}{\mathrm{~d} t^{m-1}} f(0)-\mathcal{M} g_{m-1} \in \mathrm{H}_{0}^{1}(U) .
\end{aligned}
$$

Então

$$
\frac{\mathrm{d}^{k} u}{\mathrm{~d} t^{k}} \in \mathrm{L}^{2}\left(0, T ; \mathrm{H}^{2 m+2-2 k}(U)\right), k=0, \ldots m+1 .
$$

\section{A.2.3 Uma estimativa para a solução da Equação do Calor em função dos dados iniciais e de fronteira}

O teorema enunciado nesta seção é retirado da referência [10].

Seja $\Omega_{1} \subset \mathbb{R}^{n}$ aberto conexo limitado. Denote $Q_{T}=[0, T] \times \Omega_{1}$.

Considere as normas

$$
\begin{gathered}
\|u\|_{q, r, Q_{T}}=\|u\|_{L^{r}\left([0, T], L^{q}\left(\Omega_{1}\right)\right)}=\left(\int_{0}^{T}\left(\int_{\Omega_{1}}|u(t, x)|^{q} \mathrm{~d} x\right)^{\frac{r}{q}} \mathrm{~d} t\right)^{\frac{1}{r}}, \\
\|u\|_{L^{\infty}\left([0, T], L^{2}\left(\Omega_{1}\right)\right)}=\underset{t \in[0, T]}{\operatorname{ess} \sup }\|u(t, \cdot)\|_{L^{2}\left(\Omega_{1}\right)}, \\
\|u\|_{Q_{T}}=\|u\|_{L^{\infty}\left([0, T], L^{2}\left(\Omega_{1}\right)\right)}+\left\|\nabla_{x} u\right\|_{L^{2}\left(Q_{T}\right)} .
\end{gathered}
$$

Sejam os espaços

$$
\begin{aligned}
C\left([0, T], L^{2}\left(\Omega_{1}\right)\right)= & \left\{v:[0, T] \rightarrow L^{2}\left(\Omega_{1}\right) \mid\right. \\
& \left.\lim _{\delta \rightarrow 0}\|u(t+\delta, x)-u(t, x)\|_{L^{2}\left(\Omega_{1}\right)}=0, \forall t \in[0, T]\right\},
\end{aligned}
$$




$$
V_{2}^{1,0}\left(Q_{T}\right)=L^{2}\left([0, T], H^{1}\left(\Omega_{1}\right)\right) \bigcap C\left([0, T], L^{2}\left(\Omega_{1}\right)\right)
$$

$\mathrm{e}$

$$
\stackrel{\circ}{V}_{2}^{1,0}\left(Q_{T}\right)=\left\{u \in V_{2}^{1,0}\left(Q_{T}\right) \mid u(t, x)=0, \forall(t, x) \in \partial \Omega_{1} \times[0, T]\right\}
$$

Observação A.2. Em [10] para fins didáticos, o espaço $V_{2}^{1,0}\left(Q_{T}\right)$ é apresentado como

$$
V_{2}^{1,0}\left(Q_{T}\right)=\left\{\left.u \in L^{2}\left([0, T], H^{1}\left(\Omega_{1}\right)\right)|| u\right|_{Q_{T}}<\infty\right\} \bigcap C\left([0, T], L^{2}\left(\Omega_{1}\right)\right) .
$$

Atuando em $\stackrel{\circ}{V}_{2}^{1,0}\left(Q_{T}\right)$, considere o operador

$$
\mathcal{L}(u)=\frac{\partial u}{\partial t}-\mathcal{M} u
$$

em que

$$
\mathcal{M} u=\sum_{i=1}^{n}\left[\sum_{j=1}^{n}\left(a_{i j}(t, x) u_{x_{j}}\right)_{x_{i}}+b_{i}(t, x) u-c_{i}(t, x) u_{x_{i}}\right]
$$

Os coeficientes $a_{i j}, b_{i}, c_{i}$ e $a$ do operador satisfazem a certas condições:

i ) Existem $\nu$ e $\mu$ constantes positivas tais que para todo $(t, x) \in Q_{T}$, vale

$$
\nu \sum_{i=1}^{n} \xi_{i}^{2} \leq \sum_{i=1}^{n} \sum_{j=1}^{n} a_{i j}(t, x) \xi_{i} \xi_{j} \leq \mu \sum_{i=1}^{n} \xi_{i}^{2}
$$

ii ) As normas $\left\|\sum_{i=1}^{n} b_{i}^{2}\right\|_{q, r, Q_{T}},\left\|\sum_{i=1}^{n} c_{i}^{2}\right\|_{q, r, Q_{T}} \mathrm{e}\|a\|_{q, r, Q_{T}}$ são todas limitadas por um número $\mu_{1}>0$, para quaisquer números $q$ e $r$ reais que satisfazem

$$
\begin{cases}\frac{1}{r}+\frac{n}{2 q}=1 & \\ \left.q \in] \frac{n}{2},+\infty\right], r \in[1,+\infty] & , n \geq 2 \\ q \in[1,+\infty], r \in[1,2] & , n=1,\end{cases}
$$

Teorema A.4 (LSU). Sejam as funções $f:[0, T] \times \Omega_{1} \rightarrow \mathbb{R}^{n}$ e $g:[0, T] \times \Omega_{1} \rightarrow \mathbb{R}$ tais que as normas $\|g\|_{q_{1}, r_{1}, Q_{T}} e$

$$
\|f\|_{L^{2}\left(Q_{T}\right)}=\left(\int_{0}^{T} \int_{\Omega_{1}}\left(\sum_{i=1}^{n} f_{i}^{2}(t, x)\right) \mathrm{d} x \mathrm{~d} t\right)^{\frac{1}{2}}
$$


sejam limitadas, para um par de números $r_{1}$ e $q_{1}$ que satisfazem

$$
\begin{cases}\frac{1}{r_{1}}+\frac{n}{2 q_{1}}=1+\frac{n}{4}, & \\ q_{1} \in\left[\frac{2 n}{n+2}, 2\right], r_{1} \in[1,2] & , n \geq 3 \\ \left.\left.q_{1} \in\right] 1,2\right], r_{1} \in[1,2[ & , n=2 \\ q_{1} \in[1,2], r_{1} \in\left[1, \frac{4}{3}[\right. & , n=1 .\end{cases}
$$

Seja a equação

$$
\mathcal{L}(u)=\sum_{i=1}^{n} \frac{\partial f_{i}}{\partial x_{i}}-g,
$$

em que as condições (i) e (ii) para os coeficientes são obedecidas; Então, existe c $>0$ tal que qualquer solução $u \in \stackrel{\circ}{V}_{2}^{1,0}\left(Q_{T}\right)$ que satisfaz a condição inicial

$$
\left.u\right|_{t=0}=\psi_{0} \in L_{2}\left(\Omega_{1}\right),
$$

satisfaz a desigualdade

$$
\|u\|_{Q_{T}} \leq c\left[\left\|\psi_{0}\right\|_{L^{2}\left(\Omega_{1}\right)}+\|f\|_{L^{2}\left(Q_{T}\right)}+\|g\|_{q_{1}, r_{1}, Q_{T}}\right],
$$

em que a constante $c$ depende somente de $n, \nu, \mu, \mu_{1} \mathrm{e} q$.

\section{A.3 Solução para a Equação da Onda não homogênea}

Uma solução da equação

$$
\frac{\partial^{2} u}{\partial t^{2}}-\triangle u=g
$$

é dada por

$$
u=g * \Phi_{+},
$$

em que $\Phi_{+}$, a solução fundamental da Equação da Onda, é a distribuição definida por

$$
\left\langle\Phi_{+}, \psi\right\rangle=\int_{0}^{+\infty}\left\langle\phi_{t}, \psi(t, \cdot)\right\rangle \mathrm{d} t, \quad \forall \psi \in \mathcal{C}_{c}^{\infty}\left(\mathbb{R} \times \mathbb{R}^{\mathrm{n}}\right),
$$

em que $\phi_{t}$, distribuição em $\mathbb{R}^{\mathrm{n}}$, com $n$ ímpar, é definida por:

$$
\phi_{t}(x)=\left\{\begin{array}{l}
\frac{1}{2} \mathrm{H}(t-|x|), n=1 ; \\
\frac{1}{(1.3 \ldots(n-2))}\left(t^{-1} \frac{\partial}{\partial t}\right)^{(n-3) / 2}\left[t^{n-2} \Sigma_{t}\right], n \geq 3 .
\end{array}\right.
$$

Por sua vez, na fórmula acima:

$$
\left\langle\Sigma_{t}, \psi\right\rangle=\frac{1}{w_{n}} \int_{|y|=1} \psi(t y) \mathrm{d} \sigma(y)
$$


em que $w_{n}$ é a área da esfera unitária, e $\sigma$ é a medida de área.

É possível obtermos uma fórmula fechada quando $g \in \mathcal{C}_{c}^{\infty}\left(\mathbb{R} \times \mathbb{R}^{\mathrm{n}}\right), g(t, x)=0, \forall(t, x) \in$ $\left(-\infty, 0\left[\times \mathbb{R}^{\mathrm{n}}\right.\right.$ :

$$
\begin{aligned}
u(t, x)= & g * \Phi_{+}(t, x)=\Phi_{+}(g(t-\cdot, x-\cdot))= \\
= & \int_{0}^{t}\left\langle\phi_{\tau}, g(t-\tau, x-\cdot)\right\rangle \mathrm{d} \tau= \\
= & \int_{0}^{t} \frac{1}{(1.3 \ldots(n-2))} \\
& \left(\tau^{-1} \frac{\partial}{\partial \tau}\right)^{(n-3) / 2}\left[\tau^{n-2} \frac{1}{w_{n}} \int_{|y|=1} g(\underbrace{t-\tau}_{\text {não deriva à } \tau}, x-\tau y) \mathrm{d} \sigma(y)\right] \mathrm{d} \tau .
\end{aligned}
$$

Fazendo uma mudança de variáveis a fim de trocar as posições de $\tau$ e $(t-\tau) \doteq r$, temos

$$
\begin{aligned}
u(t, x)= & \int_{0}^{t} \frac{1}{w_{n}(1.3 \ldots(n-2))} \\
& \left\{\left(\frac{1}{r} \frac{\partial}{\partial r}\right)^{(n-3) / 2}\left[r^{n-2} \int_{\partial B_{x}(1)} g(\tau, r \xi) \mathrm{d} \sigma(\xi)\right]\right\}_{r=t-\tau} \mathrm{d} \tau
\end{aligned}
$$

Para os casos particulares $n=1$ e $n=3$, temos respectivamente

$$
\begin{gathered}
u(t, x)=\frac{1}{2} \int_{0}^{t} \int_{-\tau}^{\tau} g(t-\tau, x-\xi) \mathrm{d} \xi \mathrm{d} \tau, \quad \text { para } n=1 . \\
u(t, x)=\frac{1}{4 \pi} \int_{0}^{t}(t-\tau) \int_{\partial B_{x}(1)} g(\tau,(t-\tau) \xi) \mathrm{d} \sigma(\xi) \mathrm{d} \tau, \quad \operatorname{para} n=3 .
\end{gathered}
$$

\section{A.4 Teorema de Paley Wiener}

Teorema A.5 (Paley-Wiener). Dada uma distribuição temperada $T$ em $\mathbb{R}^{n}$, as seguintes propriedades são equivalentes:

i. $O$ suporte de $T$ é compacto; $\operatorname{supp}(T) \subset\{|x| \leq A\}, A>0$.

ii. A transformada de Fourier de T pode ser estendida ao plano complexo $\mathbb{C}^{n}$ como uma função inteira $\zeta \mapsto \hat{T}(\zeta)$ tal que existem um inteiro $m \geq 0$ e uma constante $C>0$ tais que para todo $\zeta=\xi+\imath \eta$, vale

$$
|\hat{T}(\zeta)| \leq C(1+|\zeta|)^{m} \mathrm{e}^{2 \pi|\eta| A}
$$


Note que (A.11) pode ainda ser majorada, para todo $\zeta \in \mathbb{C}^{n}$ (comunicação particular de Gerson Petronilho): O termo $(1+|\zeta|)^{m}$ pode ser majorado por $2^{m} m$ ! exp [| $\left.\zeta \mid\right]$, pois como

$$
\exp [|\zeta|]=\sum_{j=0}^{\infty} \frac{|\zeta|^{j}}{j !}
$$

temos

$$
\begin{aligned}
(1+|\zeta|)^{m} & =\sum_{l=0}^{m}\left(\begin{array}{c}
m \\
l
\end{array}\right)|\zeta|^{l} \leq \sum_{l=0}^{m}\left(\begin{array}{c}
m \\
l
\end{array}\right) l ! \exp [|\zeta|] \\
& \leq m ! \exp [|\zeta|] \sum_{l=0}^{m}\left(\begin{array}{c}
m \\
l
\end{array}\right)=2^{m} m ! \exp [|\zeta|]
\end{aligned}
$$

Assim,

$$
\begin{aligned}
|\hat{T}(\zeta)| & \leq C(1+|\zeta|)^{m} \exp [2 \pi|\eta| A] \leq C 2^{m} m ! \exp [|\zeta|] \exp [2 \pi|\eta| A] \\
& \leq C 2^{m} m ! \exp [|\zeta|] \exp [2 \pi|\zeta| A]=C_{1} \exp \left[C_{2}|\zeta|\right] \leq C_{3} \exp \left[C_{3}|\zeta|\right], \quad \forall \zeta \in \mathbb{C}^{n},
\end{aligned}
$$

em que $C_{1}, C_{2}$ e $C_{3}$ são constantes convenientemente escolhidas.

\section{A.5 Teorema do Mínimo Módulo}

Definição A.1 (ordem). Uma função inteira $f$ é de ordem menor ou igual a $\rho$ se para cada $\epsilon>0$, existem uma constante $C$ e $R_{0}>0$ tal que

$$
\sup _{|z|=R}|f(z)| \leq C^{R^{(\rho+\epsilon)}}
$$

para todo $R>R_{0}$.

Definição A.2 (ordem estrita). Uma função inteira $f$ é de ordem estrita menor ou igual a $\rho$ se existem uma constante $C$ e $R_{0}>0$ tal que

$$
\sup _{|z|=R}|f(z)| \leq C^{R^{\rho}}
$$

para todo $R>R_{0}$

Uma função inteira $f$ é de ordem $\rho$ se existir

$$
\rho=\inf \{\lambda \mid f \text { é de ordem menor ou igual a } \lambda\} .
$$

Analogamente, $f$ inteira é de ordem estrita $\rho$ se existir

$$
\rho=\inf \{\lambda \mid f \text { é de ordem estrita menor ou igual a } \lambda\} .
$$




\section{Exemplo: (Exponencial)}

A função $f(z)=\mathrm{e}^{z}$ é de ordem estrita $\rho=1$, pois $\left|\mathrm{e}^{z}\right|=\mathrm{e}^{\operatorname{Re}(z)} \leq \mathrm{e}^{|z|}$.

Observação A.3. Uma função do tipo $f(\zeta)=C \exp [C|\zeta|]$ é de ordem estrita igual a um, pois para $|\zeta|>R_{0}=1$, podemos fazer a seguinte majoração:

$$
f(\zeta)=C \exp [C|\zeta|] \leq \exp [C] \exp [C|\zeta|] \leq \exp [2 C|\zeta|]=C_{1}^{|\zeta|},
$$

em que $C_{1}=\exp [2 C]$.

Essa última observação em conjunto com o Teorema de Paley-Wiener implica em que se uma função $\rho: \mathbb{R}^{\mathrm{n}} \rightarrow \mathbb{R}$ é compactamente suportada, então sua transformada de Fourier $\hat{\rho}$ é de ordem estrita igual a um.

Teorema A.6 (Mínimo módulo). Seja f uma função inteira não identicamente nula de ordem estrita igual a $\rho$. Sejam $z_{1}, z_{2}, \ldots$, os zeros de $f$, ordenando-os em ordem crescente em módulo. Seja $s>\rho$. Seja

$$
U=\mathbb{C} \backslash \bigcup_{\left|z_{n}\right|>1} \bar{B}\left(z_{n}, \frac{1}{\left|z_{n}\right|^{s}}\right)
$$

Então, existe $r_{0}>0$, que depende de $f$ tal que

$$
z \in\left\{z \in U|| z \mid>r_{0}\right\} \Rightarrow|f(z)|>\mathrm{e}^{-|z|^{\rho}} .
$$

\section{A.6 Transformada de Laplace}

Seja $T$ uma distribuição em $\mathbb{R}$, na variável $t$, com suporte na semi-reta $t \geq 0$, isto é, $T \in \mathcal{D}_{+}^{\prime}$.

Definição A.3. Dado $T \in \mathcal{D}_{+}^{\prime}$, suponha que exista $\xi_{0} \in \mathbb{R}$ tal que $t \mapsto \exp [-\xi t] T \in \mathcal{S}^{\prime}$, $\forall \xi>\xi_{0}$. Então, para qualquer função $t \mapsto \alpha(t)$ que vale $1 \mathrm{em}$ uma vizinhança do suporte de $T$ e que tem suporte limitado à esquerda, podemos calcular para dado $p=\xi+\imath \eta, \xi>\xi_{0}, \eta \in \mathbb{R}$ :

$$
\left\langle\exp \left[-\xi_{1} t\right] T, \alpha(t) \exp \left[-\left(p-\xi_{1}\right) t\right]\right\rangle,
$$

para $\xi_{0}<\xi_{1}<\xi \doteq \operatorname{Re}(p)$, já que $t \mapsto \alpha(t) \exp \left[-\left(p-\xi_{1}\right) t\right] \in \mathcal{S}$, e $\exp \left[-\xi_{1} t\right] T \in \mathcal{S}^{\prime}$. Define-se a transformada de Laplace de $T$ por

$$
\mathcal{L}(T)(p)=\langle T, \exp [-p t]\rangle \doteq\left\langle\exp \left[-\xi_{1} t\right] T, \alpha(t) \exp \left[-\left(p-\xi_{1}\right) t\right]\right\rangle,
$$

para $\operatorname{Re}(p)=\xi>\xi_{0}$

A Transformada de Laplace é injetora em seu domínio.

O seguinte teorema [12] é importante para o trabalho: 
Teorema A.7. Uma função holomorfa $\mathcal{T}(p)$ é a transformada de Laplace de uma distribuição $T \in \mathcal{D}_{+}^{\prime}$ se e somente se existir um semi-plano $\xi>c, c \in \mathbb{R}$, no qual $|\mathcal{T}(p)|$ é limitado por um polinômio em $|p|$.

Observação A.4. Note em particular que se existir $c \in \mathbb{R}$ tal que $|\mathcal{T}(p)|$ é limitado por um polinômio em $|p|$ no semi-plano $\xi>c$, então $\mathcal{L}^{-1}(\mathcal{T})$ tem suporte em $\mathbb{R}^{+}$.

\section{A.6.1 A transformada inversa}

Como existe um semi-plano em que $\mathcal{T}(p)$ é uma função inteira, há sentido em se empregar o sinal de integração:

$$
\mathcal{L}^{-1}(\mathcal{T})(t)=T(t)=\lim _{m \rightarrow+\infty} \frac{-\imath}{2 \pi} \int_{\gamma-\imath m}^{\gamma+\imath m} \mathcal{T}(p) \exp [p t] \mathrm{d} p,
$$

sendo que $\gamma>0$ está à direita de todas as singularidades de $\mathcal{T}$ (veja por exemplo [12]).

\section{A.6.2 Algumas propriedades}

Se $\operatorname{Tr}_{a}: \mathcal{D}^{\prime} \rightarrow \mathcal{D}^{\prime}, a \in \mathbb{R}$ é o operador de translação definido por

$$
\left\langle\operatorname{Tr}_{a}(T), \phi\right\rangle=\langle T, \phi(\cdot+a)\rangle, \forall \phi \text { teste, }
$$

então vale

$$
\mathcal{L}^{-1}(\exp [-a p] \mathcal{L}(T))=\operatorname{Tr}_{a}(T) .
$$

Para derivadas, vale o resultado

$$
\mathcal{L}\left(T^{(m)}\right)=p^{m} \mathcal{L}(T)
$$




\section{Bibliografia}

[1] G. Alessandrini. Stable determination of a crack from boundary measurements. Proceedings Royal Society of Edinbourg A, 127:497-516, 1988.

[2] B. Cipra. You can't always hear the shape of a drum. What's Happening in the Mathematical Sciences, 1, 1993.

[3] A. Elayyan and V. Isakov. On an inverse diffusion problem. SIAM Journal of Applied Mathematics, 57(6):1737-1748, december 1997.

[4] L. C. Evans. Partial Differential Equations, volume 19. American Mathematical Society, 1991.

[5] G. B. Folland. Introduction to Partial Differential Equations. Princeton University Press, 1995.

[6] V. Isakov. Inverse Problems for Partial Differential Equations. Springer, second edition, 2006.

[7] F. John. Plane Waves and Spherical Means Applied to Partial Differential Equations. Interscience Publishers, 1955.

[8] M. Kac. Can one hear the shape of a drum? American Mathematical Monthly, 73(4):123, 1966.

[9] A. Kawano. Projeto FAPESP proc. n.95/8964-9. Otimização no planejamento de inspeções em grupos de estruturas metálicas semelhantes sujeitas à fadiga com considerações à confiabilidade e às condições técnico-econômicas. Technical report, FAPESP, 1999.

[10] O. A. Ladyzenskaja, V. A. Solonnikov, and N. N. Ural'ceva. Linear and Quasilinear Equations of Parabolic Type. AMS, 1968. 
[11] F. G. Leitão and J. Baumeister. Topics in Inverse Problems. Publicações Matemáticas do IMPA. $25^{\circ}$ Colóquio Brasileiro de Matemática. Instituto Nacional de Matemática Pura e Aplicada (IMPA), 2005.

[12] L. Schwartz. Mathematics for the Physical Sciences. Addison-Wesley Publishing Company and Hermann- Éditeurs des Sciences et des Arts, 1966.

[13] M. Shinozuka. Development of reliability-based aircraft safety criteria. Technical report, AFFDL-TR-76-31, 1976.

[14] L. Zalcman. Analyticity and the Pompeiu problem. Archive for Rational Mechanics and Analysis, 47:237-254, 1972. 